\title{
A Leadership Competence Framework to Support the Development of Conservation Professionals
}

\author{
Simon A. Black \\ Durrell Institute of Conservation and Ecology, University of Kent, Kent, United Kingdom \\ Email: s.black@kent.ac.uk
}

How to cite this paper: Black, S. A. (2021). A Leadership Competence Framework to Support the Development of Conservation Professionals. Open Journal of Leadership, 10, 300-337.

https://doi.org/10.4236/ojl.2021.104019

Received: October 2, 2021

Accepted: December 18, 2021

Published: December 21, 2021

Copyright ( 2021 by author(s) and Scientific Research Publishing Inc. This work is licensed under the Creative Commons Attribution International License (CC BY 4.0).

http://creativecommons.org/licenses/by/4.0/

\section{(c) (i) Open Access}

\begin{abstract}
The wildlife conservation sector has been slow to examine how to better lead and manage its work and there are few empirically-based studies of conservation leadership. This study examines the importance of a wide range of leadership practices previously identified in leadership frameworks in literature. The relative importance of these practices was considered by a sample of 111 diverse conservation professionals from across the globe through an on-line survey. A principle components analysis extracted six factors which best described variances across 68 leadership items in a structure which was stable, reliable and valid. The analysis presents the first empirically-derived model of conservation leadership, including broad constructs (essentially areas of leadership competence) and clearly defined items (specific competencies) within each construct. Importantly, these constructs and the headings within challenge traditional notions of what leaders do, yet resonate strongly with a modern understanding of leadership, and in particular within the context of contemporary conservation challenges. Particularly, novel aspects in the model include: 1) an emphasis on knowledge of operational work and 2) authentic, dignified interactions with people (staff, partners and communities) and 3) a leaders' primary focus on the needs of ecosystems and species of concern. The six-factor model provides a strong basis for a future leadership development curriculum to enable thorough, integrated personal development for conservation professionals. The study offers a novel framework for guiding leadership development in the wildlife conservation sector. The detailed framework offers an important description of the specific areas of competence needed by professionals leading initiatives in complex systems of ecological, social, economic and political contexts, where personal, analytical, behavioural, and practical competencies are essential for success. This is the first such model to encompass all these areas of leadership competence.
\end{abstract}




\section{Keywords}

Leadership, Management, Factor Analysis, Competence, Capacity

Development, Conservation Professionals

\section{Introduction}

Leadership has often been mentioned as being critical to success of conservation (Dietz et al., 2004; Manolis et al., 2009; Gutierrez et al., 2011; Case et al., 2015), but the sector has only recently turned serious attention to analyzing how the approach taken by leaders affects conservation interventions and outcomes (Englefield et al., 2019; Bianco, Koss, \& Zischka, 2016; Black \& Copsey, 2014; Black, Groombridge, \& Jones, 2011; Bruyere, 2015; Haubold, 2012). In addition, there is recognition that traditional and contemporary approaches to governance (essentially leadership thinking allied to decision-making and accountability) which influences approaches to ecological interventions require new more effective consideration in the context of environmental and social challenges of the modern world (Holling \& Meffe, 1996; Acheson, 2006; Cox, 2016). Several studies of leadership and reviews of leadership literature, all within the specific context of conservation work, have been undertaken in recent years which have uncovered the broad range of topics for consideration (Manolis et al., 2009; Black, Groombridge, \& Jones, 2013; Black \& Copsey, 2014; Bruyere, 2015; Case et al., 2015; Evans et al., 2015; Black, 2019; Englefield et al., 2019; Jones \& Solomon, 2019; Webb et al., 2020). Those studies, whilst specifically pertinent to conservation leadership, have nevertheless confronted a vast array of constructs, including behaviours, capabilities, personal qualities, biases and constraints, skills, methods and approaches.

\subsection{Aspects of Leadership Relevant to Conservation}

Leadership literature is particularly diverse, covering a wide range of psychological, sociological and management theory and practice. This may be somewhat unnerving for conservation professionals less familiar with considerations of leadership; yet nevertheless much of this knowledge can relate, at a practical level, to the conservation arena (Black, 2019). Conservation leaders have the present advantage of exploring leadership beyond previous limited models of command-and-control (Holling \& Meffe, 1996), transactional leadership, behavioural theory, and transformational leadership (Bruyere, 2015) and can access wider knowledge of modern psychology and organisational systems (Black et al., 2011; Black \& Copsey, 2014).

A thorough understanding of leadership needs to recognize not only elements of a leader's behaviour, but also factors relating to the motivation of followers (Seddon, 2003). This includes consideration of less-obvious areas of interest such as the importance of leaders' influence on work design and delegation of 
authority to act (Ryan \& Deci, 2000), the engagement of followers (Van Vugt, Hogan, \& Kaiser, 2008), impact on performance (Bass et al., 2003), the relevance of leaders' vision in communicating direction and inspiring the commitment of others (Kouzes \& Posner, 2007), management of change (Higgs \& Rowland, 2011; Kouzes \& Posner, 2007), improvement of the effectiveness of organisational systems (Deming, 1994; Kilburg \& Donohue, 2011; Seddon, 2003), building trust in the workforce and with organisational partners (Rousseau, Sitkin, Burt, \& Camerer, 1998), developing social, team and organisational identity (Haslam \& Reicher, 2016), psychological safety and learning from failure (Catalano et al., 2021), support of professional development (Manolis et al., 2009; Englefield et al., 2019), developing social capital (McCallum \& O'Connell, 2009), working across different cultures (House et al., 1999), utilising personal, cultural and structural sources of power (Norbom \& Lopez, 2016), and awareness of the impact of personal charisma (Bryman, 1992).

Unsurprisingly, mainstream leadership research has developed a number of leadership scales to test many of these differing constructs. Kouzes and Posner's (2007) Leadership Practices Inventory is a particularly wide-ranging framework of transformational leadership which has been shown to measure constructs consistently in different national and cultural contexts including North America, South America, Europe, Africa, South Asia and East Asia (Posner, 2016). Other more specific scales relate to charismatic leadership (Conger et al., 1997), servant leadership (Barbuto Jr. \& Wheeler, 2006), empowering leadership (Arnold et al., 2000) and ethical leadership (Khuntia \& Suar, 2004). There are also several specialised scales which resonate with leadership challenges of the conservation sector, such as technical leadership (Thite, 1999), and leaders' implementation of evidence-based practices (Aarons, Ehrhart, \& Farahnak, 2014).

\subsection{Leadership Constructs Considered Relevant to Conservation}

In the light of the broad backdrop of leadership-related topics it is unsurprising that conservation considerations of leadership encounter a range of different issues (Black, 2019). Evans et al.'s (2015) discussion of environmental leadership, refers to Grint's (2005) typology of leadership, highlighting 1) person (behaviours, characteristics), 2) position (hierarchy, bureaucracy), 3) process (what leaders do), 4) results (resource use, sanctions, conflict resolution), and 5) purpose (direction and motivations). Four other dimensions are introduced by Bengston and Fan (1999) in the particular context of landscape conservation, namely: land stewardship, ethics, collaboration, and scientifically informed decision-making. In co-managed fisheries Gutierrez et al. (2011) motivated community leaders are critical in developing social capital and social cohesion with the programme. Mattson \& Clark (2011) examined a large multi-agency conservation project and identified the importance of leadership vision, learning, power (including money), problem solving, and community engagement. Sutton (2015) identified four elements in species reintroductions: expert leaders who "cham- 
pion" the project, individual autonomy, formal goals (and progress checks), and culturally relevant public outreach. Sjölander-Lindqvist, Johansson and Sandström (2015) suggest the relevance of similar factors in carnivore projects: gaining trust among affected people, representing stakeholders, valuing knowledge (experience, traditions, or science), collectively agreeing goals, and empowering project workers and stakeholders. Within governance of large scale landscape projects with multiple partner organisations, a collaborative, facilitative leadership approach (as opposed to a bureaucratic approach) is vital in enabling and engaging people for a common purpose (Imperial et al., 2016). At a personal level adaptability of a leader's approach is important when working with people in differing cultural contexts (Straka et al., 2018).

In addition to positive aspects, there are repeated suggestions, supported by evidence, that when conservation leaders rely on traditional power (e.g. hierarchies, reward, punishment, superior knowledge, bureaucracy) it reduces leadership effectiveness (Clark et al., 1994; Powell, 2008; Turvey, 2009; Quammen, 2012). Furthermore, those particular controls become a de facto purpose which shifts focus away from conservation (Black et al., 2011). An effective leader will avoid these traditional "command and control" approaches in order to actively counteract these negative effects (Seddon, 2003).

Recently, Englefield et al. (2019) summarised ten repeatedly arising themes across thirty articles within this developing body of conservation leadership literature, specifically:

1) collaboration and stakeholders

2) direction and motivation of others

3) decision making and empowerment

4) team culture

5) public outreach and culturally relevant community engagement

6) vision

7) adaptive management and hands-on leadership

8) sense of the bigger picture

9) networks

10) valuing knowledge including experience, traditions, science and learning

Similarly, Webb et al. (2020) highlight Stakeholder Engagement, Trust, Vision, Individual Championing (by a steadfast, inspiring leader), and Organisational excellence. Many aspects of conservation leadership including those identified in these recent reviews remain untested. To date there is no empirically-derived model that has been available to assist the professional development of conservation leaders. Despite this, several articles (Manolis et al., 2009; Black, Groombridge, \& Jones, 2011; Black, Groombridge, \& Jones, 2013; Bruyere, 2015; Straka et al., 2018) have made good attempts to explore conservation leadership across a set of relevant constructs. Case et al. (2015) suggest that Black et al. (2011) offer the most comprehensive framework: Vision and Goals, Hands-on Leadership, Details and the Big Picture, Improvement and Learning. The 43 leadership 
items identified by Black et al. (2011) were also utilised as the basis for the most recent survey of conservation leaders (Englefield et al., 2019). This suggests that, from the outset, the items proposed by Black et al. (2011) appear to offer the most suitable reference framework against which to test constructs of conservation leadership with the perceptions of experienced practitioners working in the conservation sector.

\subsection{A Focus for Examining Conservation Leadership}

This study measures the importance of leadership practices from a survey of conservation professionals. The study utilises people's direct experience; a specific approach used to avoid people's knowledge of existing frameworks causing "self-validation" of the framework within the analysis.

Research questions for the study are specifically defined as:

- Which factors are important for successful conservation leadership? These factors will be derived from the combined perceptions of experienced conservation professionals of varying backgrounds, through use of a factor analytical (principle components) design.

- Which leadership approaches make up those factors? The list of items which are retained in each factor (i.e. items selected together on the basis of the variance explained for each item) will describe the collection of leadership practices which sit within each discrete factor.

- Can these constructs be utilised to develop leaders? If the factors are well-defined, with detailed item lists, this would enable definition of a descriptive leadership development model. This framework of factors and their items can be used as an initial reference source for conservation leaders.

- Can the relative importance of leadership items be derived from suggestions of practitioners? A suitably robust and sensitive ratio scaling method will identify difference in importance rating of each item across the list of items examined in the study.

The analysis achieved by this study identifies a valid, empirical model of conservation leadership competencies which resonates with a modern understanding of leadership and the practical challenges of conservation.

\section{Methods}

The study draws on the experience of conservation professionals to derive an understanding of the relevant facets of leadership applied to conservation. Since this is the first analytical exploration of conservation leadership constructs, both the specificity of the sample (i.e. relevant people as respondents) and suitable sample size (to enable the valid application of principle components analysis) are important.

Participants were drawn from the Durrell Learning Network (an alumni group from conservation training courses run worldwide since the 1970s, and which includes employees of many different organisations; NGOs, government departments, research institutions, and corporations, in roles with responsibilities relating to biodiversity). Network members represent diverse personal, educational 
and professional backgrounds, and include a mix of ages, experience and geographic regions.

\subsection{Questionnaire Design}

A list of survey questions was initially derived from Black et al. (2011), with additional items from the most recent conservation survey (Englefield et al., 2019) which includes items from the widely-researched mainstream leadership model from Kouzes and Posner's (2007) Leadership Practices Inventory.

The questionnaire items were then reviewed for congruence with a number of established leadership scales from mainstream leadership research. In all cases the established leadership scales were shown to be accounted for within the proposed list of questionnaire items. These reference scales include Thite's (1999) 12-item technical leadership scale (which aligns with 24 items from the questionnaire), Aarons, Ehrhart and Farahnak (2014) 12-item implementation leadership scale for evidence-based practices (which aligns with 20 items), Barbuto and Wheeler's (2006) 23-item servant leadership scale (aligns with 17 questionnaire items), Conger et al.'s (1997) 20-item charismatic leadership scale (aligns with 20 items), Arnold et al.'s (2000) 37-point empowering leadership scale (37 items aligned) and Khuntia and Suar's (2004) 22-item ethical leadership scale (22 items aligned).

To further test the validity and scope of these items against previous conservation leadership research, the questions were reviewed against the constructs presented in other research papers on conservation leadership, namely the leadership skill areas identified by Bruyere (2015), principles of adaptive leadership (Manolis et al., 2009), Dietz et al.'s (2004) conservation leadership themes, and Englefield et al.'s (2019) interpersonal competencies. All aspects in these latter publications were satisfactorily covered by the proposed list of items within the survey questionnaire. This review against both management and conservation literature provided a high level of confidence that the relevant constructs of leadership were covered by the items contained in the survey instrument.

The final step in development of the survey instrument was to construct the questionnaire to follow a ratio-scaling method to measure perceptions. Ratio scaling offers the highest order in psychophysical measurement, ideal for providing measures of perception which can be examined using factor analysis (Kim \& Mueller, 1978). The ratio scale approach involves defining a reference item from the question list which assigned a standard score of 100. Respondents are asked to make numerical estimates of the importance (scores) for all other items in the survey relative to a fixed reference item score of 100 . Numerical estimation of this type is a well-established approach in psychophysical literature for measuring perception (Narens, 1996) and highly valuable in the quality of data differentiation in individual assessments of items by respondents.

\subsection{Pilot Testing}

The questionnaire was tested by eight professionals (five women, three men) 
working in Brazil, Oman, Cambodia, Indonesia, India, Mauritius and the UK. These professionals had 5 to 20 years' experience in various roles (field manager, supervisor, researcher, senior manager, consultant). Six had English as a second language (being nationals from Brazil, India, Germany, Oman, Netherlands, and Venezuela). The pilot respondents had high levels of competence to make reasonable comments on the clarity of terminology used in the questionnaire and its suitability for the intended sample of international professionals.

Feedback from the pilot group participants included suggestions for the refinement of instructions, changes to layout and amendment to the phraseology in specific questions. Several questions were split, with duplicates removed. The pilot group agreed that the item "Establish budgets and manage costs" was the most neutral and commonly-encountered item (in normal work situations) included in the list of questions, and therefore the most suitable for use as a reference item. The final questionnaire included 70 leadership items. To reduce the influence of response fatigue, the items were organised in Qualtrics in blocks of five per screen, and randomised when accessed on-line by each new respondent. Within blocks, the question order was also randomised. Demographic questions drawn from Englefield et al. (2019) covered gender, age and professional status.

\subsection{Procedure}

Invitations were emailed to 281 conservation professionals with an on-line link to the Qualtrics site (https://www.qualtrics.com/) with participation anonymously monitored by the software to allow automated email reminders. A link was also provided on Durrell network blogs to encourage participation. The on-line questionnaire was accessed by 111 people (39.5\% response). Participants were asked to estimate the importance of 70 items based upon their personal leadership experience or from their own observation of leaders.

\subsection{Survey Sample}

Respondents worked in Non-Governmental Organisations (40 cases), government (2), research institutions (8), Multi-Agency project (1), commercial organisation (1) "other" (8), or unspecified (51). Twenty nationalities were reported: UK (21 people), USA (7), India (3), Brazil (2), Canada (2), France (2), Mauritius (2), Seychelles (2), Germany (2), and one each from Albania, Algeria, Australia, Belize, Ethiopia, Italy, Madagascar, Morocco, Netherlands, Spain and Zimbabwe. Participants had a range of ages and experience with 25 women, 35 men, and 51 gender unspecified (Table 1).

For the principle components analysis (PCA) approach applied in the study, a sample of at least 50 participants (i.e. respondents) is needed for clear and stable patterns from the factor analysis (Barrett \& Kline, 1981) and usually at least 100 participants (Dancey \& Reidy, 2011). A sample ideally larger in size than the number of items measured in the survey instrument is also recommended (Barrett \& Kline, 1981). The sample of 111 participants therefore fulfils these requirements for minimum sample size. 
Table 1. Self-reported profile of professionals participating in the survey.

\begin{tabular}{|c|c|}
\hline a) Region/Continent & No. of participants \\
\hline North America & 9 \\
\hline Europe & 29 \\
\hline Africa and Indian Ocean & 10 \\
\hline Asia & 3 \\
\hline South America \& Caribbean & 3 \\
\hline Oceania and Pacific & 1 \\
\hline Not stated & 56 \\
\hline b) Experience profile & No. of participants \\
\hline 5 years or less & 11 \\
\hline $6-10$ years & 13 \\
\hline $11-15$ years & 14 \\
\hline $16-20$ years & 4 \\
\hline more than 20 years & 17 \\
\hline not stated & 52 \\
\hline c) Age profile & No. of participants \\
\hline $20-29$ years & 7 \\
\hline $30-39$ years & 16 \\
\hline $40-49$ & 20 \\
\hline $50-59$ & 13 \\
\hline $60-69$ & 4 \\
\hline not stated & 51 \\
\hline
\end{tabular}

\subsection{Factor Analysis of the Survey Responses}

Analysis was conducted using the software package SPSS. Factor analysis was applied to the 70 numerically estimated items (excluding the reference). A scree plot of factor eigenvalues was used to identify the number of valid factors extracted (Rummel, 1970). Since the factor structure accounts for the maximum amount of variance in items but not the maximum correlations between factors and items, it is common to apply a rotation procedure (Harman, 1967: pp. 93-94). Rotation maximises the correlations of items giving a more readily interpreted set of factors (Comrey, 1973). The review of literature demonstrates that the construct of leadership is comprised of interrelated rather than unrelated factors, so oblique rotation is the most suitable method (Thurstone, 1947).

\subsection{Tests of Reliability and Validity}

Several tests of reliability and validity assessed the stability and relevance of the factors.

Obliquel orthogonal rotation. A tentative test for construct validity was based 
on repeat analysis with varimax rotation to determine if the factor structure is maintained.

Cronbach's alpha for reliability of each factor. A further test of reliability (Cronbach, 1951) of each of the factors follows Nunnally's (1967) suggestion that in exploratory studies, such as this present study, Cronbach's alpha of 0.6 is sufficient across factors and this is reported in the discussion of results.

Construct validity through unifactorial testing. The discrete sets of items in each factor are subjected to an individual factor analysis (Nunnally, 1967). If all items are retained as a single factor, that factor is a valid construct ("unifactorial determination").

Sampling adequacy. The Kaiser-Meyer-Olkin statistic (KMO) compares the number of variables and correlation between them to evaluate sampling adequacy with scores above 0.8 being meritorious and 0.9 being very good (Dziuban \& Shirkey, 1974; Kim \& Mueller, 1978: p. 54).

Content validity. Content validity assessment involves definition of factor labels (descriptors) by informed independent observers using Rummel's (1970) descriptive perspective method, describing relationships between items in a factor using few words for easy recall with a view to their future use.

\section{Results}

The sample of 111 people was considered representative of conservation professionals and appropriate for factor analysis (Table 1).

Where stated by the respondent, fewer participants were from Asia (6\% of stated responses), South America (6\%) and Oceania (2\%) than the proportion from Europe (53\% of those stating that region, including UK 14\%), North America (16\%), and Africa (18\%) although 56 people from the sample did not disclose nationality. Despite a skew towards UK and western countries, the mix of 20 nationalities which were reported by respondents compares well with other surveys. Englefield et al. (2019) involved 130 respondents from 16 nationalities $(44.6 \%$ UK, 30.7\% North America, 3.8\% Europe, 4.6\% Africa; 3.9\% Asia, 1.6\% South America, 6.9\% Oceania and Pacific) whilst the study by Bruyere (2015) involved 103 people from 15 countries.

\subsection{Reliability of the Survey Instrument}

Cronbach's alpha was 0.99 across all 70 items. A split-halves test returned reliabilities of 0.99 and 0.98 indicating the questionnaire was a reliable instrument.

\subsection{Correlations across 70 Leadership Items}

Most correlations across the 70 leadership items were significant with Pearson's Correlation Coefficient across a two tailed test (for interrelated constructs). Three items had low correlations across the data: "Achieve a good personal reputation in conservation through successes" (v56), "Provide training on a just-in-time basis" (v26), and "Have a clear philosophy of leadership" (v55), 
however each had higher correlations with several items, so were not removed from the factor analysis (Arnold et al., 2000).

\subsection{Factor Analysis of 70 Leadership Items}

Initial factor analysis (principle components) identified nine factors with eigenvalues greater than 1 after which variance explained by subsequent factors was less than 1.5\% (Table 2). Further to this, a scree plot (Figure 1) identified which factors to consider as "scree" (i.e. to be dropped from the analysis) which resulted in just six factors being retained after which eigenvalues level out, this being a well established method for identifying the less relevant "scree" factors which are rejected (Cattell, 1978). Factor analysis was thereafter repeated to extract six factors, with an oblique rotation (oblimin in SPSS) being applied to optimise the model (Rummel, 1970). Items in the subsequent initial pattern matrix had sufficient loadings on at least one factor. Two items in the initial structure matrix, v47 (0.520) and v51 (0.426), had insufficient loadings with any factors (less than 0.58 or one-third of item variance) so these two items (v47, v51) were removed from the analysis (Comrey, 1973).

Table 2. Eigenvalues for initial factor solution (70 items, Figure 1) and variance explained.

\begin{tabular}{|c|c|c|c|}
\hline Component & Eigenvalue: Total & $\%$ of Variance & Cumulative \% \\
\hline 1 & 47.447 & 67.782 & 67.782 \\
\hline 2 & 2.895 & 4.136 & 71.918 \\
\hline 3 & 2.269 & 3.241 & 75.159 \\
\hline 4 & 1.915 & 2.735 & 77.894 \\
\hline 5 & 1.598 & 2.283 & 80.177 \\
\hline 6 & 1.542 & 2.203 & 82.380 \\
\hline 7 & 1.326 & 1.894 & 84.274 \\
\hline 8 & 1.160 & 1.658 & 85.932 \\
\hline 9 & 1.063 & 1.519 & 87.451 \\
\hline 10 & 0.968 & 1.383 & 88.834 \\
\hline 11 & 0.863 & 1.233 & 90.066 \\
\hline 12 & 0.804 & 1.149 & 91.216 \\
\hline 13 & 0.648 & 0.926 & 92.141 \\
\hline 14 & 0.594 & 0.849 & 92.990 \\
\hline 15 & 0.494 & 0.705 & 93.696 \\
\hline 16 & 0.461 & 0.658 & 94.354 \\
\hline 17 & 0.392 & 0.560 & 94.914 \\
\hline 18 & 0.356 & 0.508 & 95.422 \\
\hline 19 & 0.330 & 0.472 & 95.894 \\
\hline 20 & 0.309 & 0.441 & 96.335 \\
\hline 21 & 0.263 & 0.376 & 96.711 \\
\hline
\end{tabular}




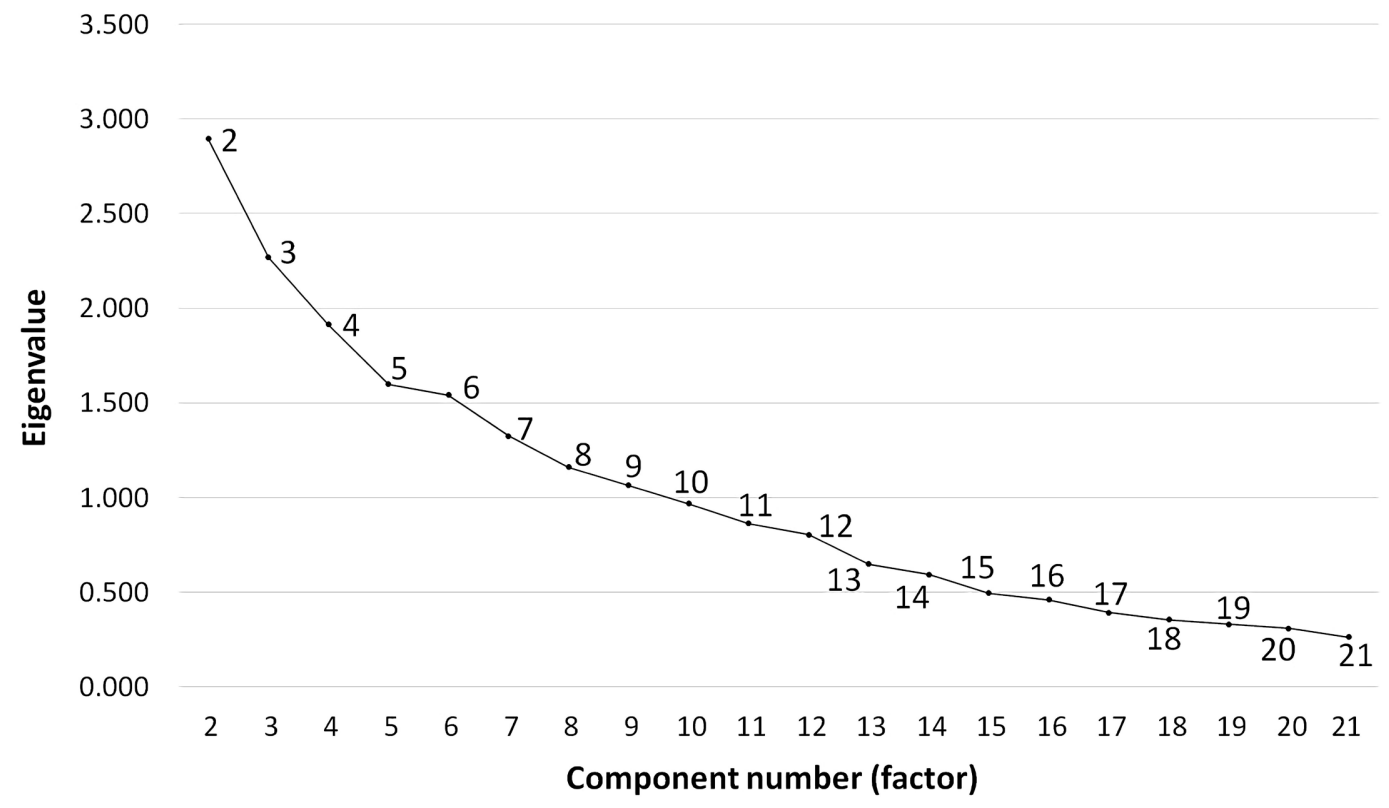

Figure 1. Scree plot of eigenvalues (factors 2 - 21) levels at factors 7, 8, 9, 10, 11, 12 for Cattel's (1978) criterion of "more than three-in-a row" (note: Factor 1 eigenvalue 67.8).

\subsection{Revised Six Factor Solution for 68 Items}

With items v47 and v51 removed from the analysis. A repeat factor analysis was applied to the final 68 items. The scree plot confirmed the six-factor structure after oblimin rotation (38 iterations) and factors correlated less than 0.539 (Table 3) so each is considered an independent construct (Arnold et al., 2000). The pattern matrix (Table 4) defined the item clusters (Rummel, 1970). All loadings in the structure matrix (Table 5) exceed 0.58 so all 68 items are retained (Comrey, 1973).

\subsection{Reliability of the 6-Factor Structure}

Cronbach's alpha as a test of reliability of each factor.

Coefficient alpha calculation for the item set in each of the six factors, revealed values of $0.98,0.98,0.96,0.88,0.98$, and 0.92 for factors 1 to 6 respectively (Supplementary Table S2). All of the six factors were determined to be reliable.

Sampling adequacy.

All factors (Tables S1(a)-(f)) have a KMO rated as "meritorious" to "very good", $(0.930,0.935,0.920,0.824,0.931,0.868)$, so the number of variables and correlation between them is well-suited to factor analysis (Cerny \& Kaiser, 1977; Kim \& Mueller, 1978).

\subsection{Content Validity of the Six Factors}

Factor items in the pattern matrix (Table 4) were reviewed by six independent experts; an HR professional, an occupational psychologist, a CEO of a conservation NGO, a conservation academic, a leadership researcher, and a charity director. All items are highly correlated with the factor, but the factor is best explained 
Table 3. Correlations between factors in the six-factor model from 68 items.

\begin{tabular}{ccccccc}
\hline \multicolumn{7}{c}{ Component Correlation Matrix } \\
\hline Component & 1 & 2 & 3 & 4 & 5 & 6 \\
\hline 1 & 1.000 & & & & & \\
2 & 0.539 & 1.000 & & & & \\
3 & 0.352 & 0.371 & 1.000 & & & \\
4 & 0.410 & 0.373 & 0.353 & 1.000 & & \\
5 & 0.517 & 0.427 & 0.348 & 0.343 & 1.000 & \\
6 & 0.471 & 0.414 & 0.282 & 0.345 & 0.457 & 1.000 \\
\hline
\end{tabular}

Extraction Method: Principal Component Analysis. Rotation Method: Oblimin with Kaiser Normalization.

Table 4. Pattern Matrix of the revised six factor solution for 68 leadership items have under oblimin rotation (v47 and v51 removed) presented in order of factor loading.

\begin{tabular}{|c|c|c|c|c|c|c|}
\hline & \multicolumn{6}{|c|}{$\begin{array}{l}\text { Pattern Matrix } \\
\text { Component }\end{array}$} \\
\hline & 1 & 2 & 3 & 4 & 5 & 6 \\
\hline 13 Skills & 0.690 & -0.209 & -0.016 & 0.384 & -0.013 & 0.137 \\
\hline 49 Keep promises & 0.679 & 0.063 & 0.064 & -0.112 & 0.155 & 0.265 \\
\hline 3 Identify threats & 0.670 & 0.179 & 0.221 & 0.048 & -0.142 & 0.162 \\
\hline 15 Empower & 0.600 & 0.196 & 0.211 & 0.042 & 0.133 & 0.021 \\
\hline 60 Complexity & 0.588 & 0.135 & -0.137 & 0.204 & 0.203 & 0.141 \\
\hline 5 Planning & 0.570 & -0.003 & 0.134 & 0.052 & 0.167 & 0.303 \\
\hline 7 Stakeholders & 0.568 & -0.015 & 0.075 & 0.035 & 0.439 & -0.059 \\
\hline 59 Use knowledge & 0.551 & 0.180 & 0.076 & 0.332 & -0.204 & 0.269 \\
\hline 27 Open minded & 0.530 & 0.301 & -0.004 & 0.027 & 0.257 & 0.087 \\
\hline 61 Note implication & 0.529 & 0.061 & 0.141 & 0.061 & 0.156 & 0.267 \\
\hline 50 Dignity \& respect & 0.498 & 0.075 & 0.095 & 0.077 & 0.238 & 0.209 \\
\hline 16 Know people & 0.454 & 0.293 & 0.052 & 0.077 & 0.336 & 0.036 \\
\hline 21 Progress meetings & 0.447 & 0.215 & 0.249 & 0.087 & 0.383 & -0.133 \\
\hline 29 Open to challenge & 0.440 & 0.313 & 0.156 & 0.032 & 0.062 & 0.216 \\
\hline 30 Share and learn & 0.416 & 0.273 & 0.238 & 0.085 & 0.024 & 0.229 \\
\hline 31 Expectations & 0.401 & 0.233 & 0.066 & 0.247 & -0.003 & 0.259 \\
\hline 8 Current state & -0.003 & 0.766 & 0.232 & 0.061 & -0.058 & 0.056 \\
\hline 26 Provide training & 0.096 & 0.723 & -0.514 & -0.114 & -0.021 & 0.329 \\
\hline 18 Involve people & 0.090 & 0.683 & 0.107 & 0.135 & -0.020 & 0.179 \\
\hline 39 Funding strategy & -0.114 & 0.647 & 0.204 & 0.178 & -0.064 & 0.178 \\
\hline 37 Influence & -0.011 & 0.569 & 0.289 & 0.114 & 0.139 & 0.128 \\
\hline 36 Experimentation & 0.211 & 0.547 & 0.216 & 0.049 & 0.061 & 0.126 \\
\hline
\end{tabular}




\section{Continued}

\begin{tabular}{|c|c|c|c|c|c|c|}
\hline 10 Governance & 0.119 & 0.528 & 0.088 & 0.121 & 0.230 & 0.140 \\
\hline 19 Delegate control & 0.165 & 0.517 & 0.200 & 0.204 & 0.143 & -0.032 \\
\hline 57 Seek experience & 0.034 & 0.508 & -0.043 & 0.367 & 0.206 & 0.169 \\
\hline 12 Alignment & 0.207 & 0.491 & 0.329 & 0.253 & -0.228 & 0.155 \\
\hline 14 Prioritise & 0.364 & 0.486 & 0.202 & 0.162 & 0.154 & -0.147 \\
\hline 28 Consider risk & 0.326 & 0.480 & 0.015 & 0.076 & 0.188 & 0.095 \\
\hline 32 System not people & 0.179 & 0.473 & -0.006 & 0.020 & 0.299 & -0.214 \\
\hline 4 Goals & 0.272 & 0.392 & 0.364 & 0.174 & -0.006 & 0.107 \\
\hline 25 Training requests & 0.344 & 0.356 & 0.303 & -0.236 & 0.269 & 0.046 \\
\hline 11 Hands-on & 0.249 & 0.345 & 0.335 & 0.117 & 0.235 & -0.078 \\
\hline 23 Listen & 0.271 & 0.332 & 0.199 & 0.135 & 0.191 & 0.140 \\
\hline 24 Expect change & 0.134 & 0.133 & 0.696 & 0.201 & -0.113 & 0.069 \\
\hline 40 Performance means & 0.351 & 0.102 & 0.672 & -0.200 & 0.097 & 0.053 \\
\hline 34 Drive improvement & 0.013 & 0.094 & 0.542 & 0.328 & 0.050 & 0.238 \\
\hline 35 Capable v neglect & -0.099 & 0.230 & 0.541 & -0.032 & 0.434 & 0.135 \\
\hline 38 organ dynamics & -0.245 & 0.192 & 0.450 & 0.323 & 0.337 & 0.221 \\
\hline 9 Staff aims & 0.125 & 0.146 & 0.391 & 0.228 & 0.105 & 0.322 \\
\hline 2 Purpose & 0.335 & 0.045 & 0.361 & 0.132 & 0.235 & 0.223 \\
\hline 41 Resource needs & 0.060 & 0.041 & 0.342 & 0.272 & 0.223 & 0.249 \\
\hline 22 Clarify & 0.231 & 0.234 & 0.325 & 0.065 & 0.141 & 0.253 \\
\hline 56 Reputation & 0.025 & -0.034 & -0.083 & 0.914 & -0.071 & 0.006 \\
\hline 45 Community involve & -0.121 & 0.073 & 0.257 & 0.706 & 0.156 & 0.047 \\
\hline 6 Performance $v$ aims & 0.263 & 0.213 & 0.409 & 0.412 & 0.002 & -0.194 \\
\hline 1 Vision & 0.246 & -0.166 & 0.148 & 0.406 & 0.225 & 0.342 \\
\hline 62 Personal strength & 0.377 & 0.312 & -0.122 & 0.385 & 0.136 & 0.037 \\
\hline 55 Leadership ethos & -0.001 & -0.249 & -0.030 & 0.023 & 0.870 & 0.212 \\
\hline 46 Future trends & 0.052 & 0.141 & 0.260 & -0.070 & 0.644 & 0.088 \\
\hline 64 Emotional impact & 0.063 & 0.100 & 0.181 & 0.047 & 0.598 & 0.163 \\
\hline 53 Support decisions & -0.084 & 0.234 & 0.122 & 0.192 & 0.566 & 0.194 \\
\hline 68 Team talents & 0.253 & 0.247 & -0.034 & 0.343 & 0.553 & -0.243 \\
\hline 67 Adapt style & 0.228 & 0.062 & -0.008 & 0.148 & 0.548 & 0.200 \\
\hline 66 Spot potential & 0.264 & 0.093 & -0.183 & 0.180 & 0.527 & 0.234 \\
\hline 52 What can we learn & 0.099 & -0.065 & 0.300 & 0.156 & 0.526 & 0.192 \\
\hline 48 Confident in team & 0.240 & 0.181 & -0.088 & 0.184 & 0.500 & 0.172 \\
\hline 54 Recog committed & 0.177 & 0.374 & 0.121 & -0.190 & 0.488 & 0.175 \\
\hline 69 Networks & 0.062 & 0.391 & -0.141 & 0.406 & 0.478 & -0.005 \\
\hline 33 Manage morale & 0.179 & 0.104 & 0.330 & 0.042 & 0.443 & 0.173 \\
\hline
\end{tabular}




\section{Continued}

\begin{tabular}{lcccccc}
\hline 70 Develop self & 0.368 & 0.183 & -0.091 & 0.299 & 0.436 & -0.008 \\
17 Cultural sense & 0.243 & 0.069 & 0.093 & 0.018 & 0.424 & 0.322 \\
65 Provide expertise & 0.367 & 0.094 & 0.054 & -0.064 & 0.409 & 0.297 \\
42 Partnerships & 0.148 & 0.149 & 0.094 & 0.193 & 0.361 & 0.313 \\
43 Flexibility & 0.001 & 0.090 & -0.056 & -0.070 & 0.269 & 0.769 \\
58 Passion for work & 0.188 & 0.086 & -0.089 & 0.149 & 0.009 & 0.707 \\
20 What matters & 0.185 & 0.051 & 0.122 & 0.071 & 0.000 & 0.644 \\
63 Humility & -0.086 & 0.065 & 0.176 & 0.255 & 0.265 & 0.498 \\
44 Seek advice & 0.374 & 0.079 & 0.178 & -0.116 & 0.213 & 0.409
\end{tabular}

Extraction Method: Principal Component Analysis. Rotation Method: Oblimin with Kaiser Normalization. ${ }^{a}$ Rotation converged in 38 iterations.

Table 5. Structure Matrix for 68 items showing acceptable loadings on all factors.

\begin{tabular}{|c|c|c|c|c|c|c|}
\hline & \multicolumn{6}{|c|}{$\begin{array}{l}\text { Structure Matrix } \\
\text { Component }\end{array}$} \\
\hline & 1 & 2 & 3 & 4 & 5 & 6 \\
\hline 49 Keep promises & 0.894 & 0.586 & 0.415 & 0.357 & 0.638 & 0.661 \\
\hline 15 Empower & 0.876 & 0.679 & 0.562 & 0.489 & 0.624 & 0.520 \\
\hline 27 Open minded & 0.876 & 0.740 & 0.418 & 0.473 & 0.707 & 0.587 \\
\hline 60 Complexity & 0.868 & 0.622 & 0.303 & 0.566 & 0.652 & 0.599 \\
\hline 3 Identify threats & 0.867 & 0.647 & 0.537 & 0.476 & 0.449 & 0.566 \\
\hline 5 Planning & 0.865 & 0.570 & 0.495 & 0.494 & 0.663 & 0.702 \\
\hline 16 Know people & 0.852 & 0.743 & 0.474 & 0.518 & 0.756 & 0.566 \\
\hline 61 Note implication & 0.843 & 0.598 & 0.501 & 0.497 & 0.648 & 0.674 \\
\hline 59 Use knowledge & 0.832 & 0.653 & 0.459 & 0.675 & 0.421 & 0.646 \\
\hline 50 Dignity \& respect & 0.825 & 0.595 & 0.466 & 0.496 & 0.682 & 0.636 \\
\hline 21 Progress meetings & 0.822 & 0.689 & 0.613 & 0.524 & 0.762 & 0.442 \\
\hline 29 Open to challenge & 0.810 & 0.736 & 0.521 & 0.480 & 0.587 & 0.636 \\
\hline 30 Share and learn & 0.802 & 0.722 & 0.588 & 0.529 & 0.572 & 0.645 \\
\hline 7 Stakeholders & 0.801 & 0.496 & 0.419 & 0.420 & 0.738 & 0.437 \\
\hline 13 Skills & 0.788 & 0.352 & 0.319 & 0.627 & 0.444 & 0.498 \\
\hline 70 Develop self & 0.780 & 0.642 & 0.362 & 0.634 & 0.773 & 0.519 \\
\hline 31 Expectations & 0.771 & 0.672 & 0.453 & 0.610 & 0.530 & 0.647 \\
\hline 2 Purpose & 0.767 & 0.601 & 0.687 & 0.572 & 0.700 & 0.655 \\
\hline 62 Personal strength & 0.748 & 0.687 & 0.320 & 0.673 & 0.571 & 0.504 \\
\hline 44 Seek advice & 0.734 & 0.563 & 0.487 & 0.344 & 0.649 & 0.725 \\
\hline 25 Training requests & 0.707 & 0.700 & 0.580 & 0.254 & 0.644 & 0.483 \\
\hline 22 Clarify & 0.691 & 0.669 & 0.637 & 0.498 & 0.612 & 0.638 \\
\hline
\end{tabular}




\section{Continued}

\begin{tabular}{|c|c|c|c|c|c|c|}
\hline 18 Involve people & 0.625 & 0.887 & 0.484 & 0.520 & 0.484 & 0.572 \\
\hline 8 Current state & 0.513 & 0.872 & 0.532 & 0.427 & 0.394 & 0.432 \\
\hline 36 Experimentation & 0.694 & 0.838 & 0.568 & 0.481 & 0.554 & 0.558 \\
\hline 10 Governance & 0.669 & 0.826 & 0.488 & 0.525 & 0.653 & 0.586 \\
\hline 37 Influence & 0.577 & 0.825 & 0.621 & 0.516 & 0.574 & 0.543 \\
\hline 14 Prioritise & 0.774 & 0.822 & 0.580 & 0.566 & 0.608 & 0.409 \\
\hline 28 Consider risk & 0.763 & 0.809 & 0.427 & 0.492 & 0.637 & 0.564 \\
\hline 57 Seek experience & 0.629 & 0.804 & 0.406 & 0.684 & 0.628 & 0.603 \\
\hline 19 Delegate control & 0.657 & 0.804 & 0.563 & 0.573 & 0.574 & 0.452 \\
\hline 12 Alignment & 0.647 & 0.786 & 0.638 & 0.613 & 0.361 & 0.532 \\
\hline 4 Goals & 0.730 & 0.780 & 0.695 & 0.595 & 0.537 & 0.558 \\
\hline 39 Funding strategy & 0.430 & 0.774 & 0.494 & 0.484 & 0.366 & 0.482 \\
\hline 23 Listen & 0.740 & 0.741 & 0.571 & 0.555 & 0.652 & 0.595 \\
\hline 11 Hands-on & 0.686 & 0.715 & 0.651 & 0.520 & 0.632 & 0.424 \\
\hline 26 Provide training & 0.403 & 0.669 & -0.167 & 0.120 & 0.270 & 0.480 \\
\hline 32 System not people & 0.494 & 0.614 & 0.284 & 0.297 & 0.501 & 0.209 \\
\hline 24 Expect change & 0.507 & 0.519 & 0.844 & 0.536 & 0.356 & 0.401 \\
\hline 40 Performance means & 0.635 & 0.529 & 0.811 & 0.271 & 0.511 & 0.425 \\
\hline 34 Drive improvement & 0.527 & 0.544 & 0.782 & 0.659 & 0.507 & 0.572 \\
\hline 35 Capable v neglect & 0.491 & 0.607 & 0.769 & 0.400 & 0.720 & 0.524 \\
\hline 38 Organ dynamics & 0.429 & 0.583 & 0.729 & 0.646 & 0.661 & 0.578 \\
\hline 9 Staff aims & 0.641 & 0.622 & 0.697 & 0.619 & 0.593 & 0.678 \\
\hline 41 Resource needs & 0.547 & 0.500 & 0.622 & 0.595 & 0.597 & 0.586 \\
\hline 56 Reputation & 0.319 & 0.262 & 0.214 & 0.860 & 0.215 & 0.264 \\
\hline 45 Community involve & 0.401 & 0.452 & 0.558 & 0.844 & 0.477 & 0.408 \\
\hline 1 Vision & 0.653 & 0.411 & 0.491 & 0.693 & 0.629 & 0.675 \\
\hline 6 Performance $\mathrm{v}$ aims & 0.600 & 0.580 & 0.672 & 0.677 & 0.424 & 0.277 \\
\hline 55 Leadership ethos & 0.414 & 0.207 & 0.248 & 0.291 & 0.858 & 0.506 \\
\hline 46 Future trends & 0.566 & 0.551 & 0.555 & 0.348 & 0.838 & 0.515 \\
\hline 67 Adapt style & 0.698 & 0.554 & 0.395 & 0.520 & 0.832 & 0.632 \\
\hline 64 Emotional impact & 0.586 & 0.541 & 0.511 & 0.436 & 0.827 & 0.575 \\
\hline 53 Support decisions & 0.548 & 0.628 & 0.499 & 0.549 & 0.819 & 0.611 \\
\hline 48 Confident in team & 0.721 & 0.631 & 0.351 & 0.549 & 0.812 & 0.627 \\
\hline 66 Spot potential & 0.707 & 0.557 & 0.258 & 0.521 & 0.809 & 0.649 \\
\hline 54 Recog committed & 0.678 & 0.724 & 0.474 & 0.293 & 0.796 & 0.605 \\
\hline 52 What can we learn & 0.597 & 0.462 & 0.603 & 0.525 & 0.795 & 0.591 \\
\hline 33 Manage morale & 0.679 & 0.600 & 0.649 & 0.483 & 0.789 & 0.611 \\
\hline
\end{tabular}




\section{Continued}

\begin{tabular}{lllllll}
\hline 68 Team talents & 0.687 & 0.634 & 0.392 & 0.634 & 0.785 & 0.341 \\
65 Provide expertise & 0.762 & 0.585 & 0.421 & 0.384 & 0.771 & 0.689 \\
69 Networks & 0.635 & 0.726 & 0.335 & 0.690 & 0.765 & 0.506 \\
17 Cultural sense & 0.691 & 0.556 & 0.449 & 0.433 & 0.765 & 0.691 \\
42 Partnerships & 0.675 & 0.619 & 0.483 & 0.574 & 0.743 & 0.703 \\
43 Flexibility & 0.503 & 0.477 & 0.263 & 0.302 & 0.616 & 0.890 \\
58 Passion for work & 0.603 & 0.507 & 0.265 & 0.475 & 0.487 & 0.863 \\
20 What matters & 0.588 & 0.490 & 0.413 & 0.432 & 0.479 & 0.812 \\
63 Humility & 0.487 & 0.498 & 0.492 & 0.569 & 0.625 & 0.743 \\
\hline
\end{tabular}

Extraction Method: Principal Component Analysis. Rotation Method: Oblimin with Kaiser Normalization.

by the first items in order (see Table 7). Each expert offered a title definition for each factor, based upon an assessment of the content of the items within each factor. The experts' suggestions were collated and amalgamated into a cogent theme ("factor title"). Factor titles were defined after consideration of existing leadership scales in literature. Each factor was interpreted as a short "memorable title" (in parentheses below) to meet Rummel's (1970) criteria:

1) Conserves by engaging followers (Serve Biodiversity through Followers)

2) Understands operational work (Understand Operational Work)

3) Effectiveness through a sense of reality (Sense of Reality)

4) Provides compelling visionary leadership (Visionary Leadership)

5) Provides ethical participative leadership (Authentic Engagement)

6) Places purpose before ego (Purpose before Ego)

\subsection{Construct Validity of the Factor Structure}

The overall framework was notionally compared to existing mainstream leadership models to examine whether reasonable constructs of leadership were present in the collection of items under each factor. The models used for comparison were Kouzes and Posner's (2007) Five Practices of Exemplary Leadership model (which gave rise to the popular Leadership Practices Inventory assessment tool), Bass's (2000) Full Range of Leadership model (the basis of the Multifactor Leadership Questionnaire), and Covey's (1999) Principle Centred Leadership model (which includes the 7 habits of leadership compared with Deming' (1994) highly influential "14 Points for Management"). The Six Factors of Conservation Leadership derived from this analysis align satisfactorily with these established leadership models, indicating construct validity of the factors (Table 6).

Unifactorial test of construct validity of each factor.

All six factors were determined as strongly unifactorial (Supplementary Table S1). Each factor accounts for most variance in all of its items on repeat factor analysis of each specific set of factor items. This confirms that each factor as a 
Table 6. Comparison of the six factor constructs with established leadership models.

\begin{tabular}{|c|c|c|c|}
\hline $\begin{array}{l}\text { Six Factors of } \\
\text { Conservation } \\
\text { Leadership }\end{array}$ & $\begin{array}{c}\text { Covey's (1999) } \\
\text { Principled Centred } \\
\text { Leadership (Seven Habits) }\end{array}$ & $\begin{array}{l}\qquad \text { Bass (2000) } \\
\text { Full Range of Leadership Model }\end{array}$ & $\begin{array}{l}\text { Kouzes and Posner } \\
\text { (2007) Exemplary } \\
\text { Leadership }\end{array}$ \\
\hline $\begin{array}{l}\text { 1) Serve biodiversity } \\
\text { through followers }\end{array}$ & - Synergize: creative cooperation & $\begin{array}{l}\text { - "Ideal Influence Attributes" \& } \\
\text { "Behaviours" } \\
\text { - "Contingent Reward" } \\
\text { opposite to: } \\
\text { • "Passive" }\end{array}$ & "Enable Others to Act" \\
\hline $\begin{array}{l}\text { 2) Understand } \\
\text { operational work }\end{array}$ & $\begin{array}{l}\text { - Sharpen the saw: continuous } \\
\text { improvement }\end{array}$ & $\begin{array}{l}\text { - "Intellectual Stimulation" } \\
\text { (in part - see below) and } \\
\text { - "Active Management" }\end{array}$ & "Challenge the Process" \\
\hline 3) Sense of reality & $\begin{array}{l}\text { Put first things first: manage } \\
\text { time/priorities around goals \& roles }\end{array}$ & - "Intellectual Stimulation" (in part) & "Challenge the Process" \\
\hline 4) Visionary leadership & $\begin{array}{l}\text { - Be proactive: self-awareness, } \\
\text { personal vision \& responsibility } \\
\text { - Seeks first to understand: empathic } \\
\text { communication }\end{array}$ & $\begin{array}{l}\text { - "Inspirational Motivation" } \\
\text { and part of } \\
\text { - "Idealised Influence Behaviours" }\end{array}$ & "Inspire a Shared Vision" \\
\hline 5) Authentic engagement & - Think win-win: seek mutual benefit & • "Individual Consideration" & "Model the Way" \\
\hline 6) Purpose before ego & $\begin{array}{l}\text { "Begin with an end in mind": } \\
\text { leadership \& mission }\end{array}$ & $\begin{array}{l}\text { "Idealised Influence" (in part see } \\
\text { above) }\end{array}$ & "Encourage the Heart" \\
\hline
\end{tabular}

good descriptor of its defined set of items, and a clearly defined construct of conservation leadership.

\subsection{Importance Scores on Each Item}

The relative importance of items was derived from ratio scores of importance given for each item by the 111 respondents. The natural log of all responses on each item was calculated, then the mean of those log scores for the item was calculated, with the final item importance score calculated as the exponent of that mean of natural logs from all respondent scores (Lodge, 1981). Since the scores are converted to natural logs, the distribution moves towards a normal distribution (Lodge, 1981), as shown in supplementary Figure S1. The highest importance scores (covering the top $10 \%$ of items in the questionnaire framework) were: v50 "Treat others with dignity" (187 points); v2 "Establish a shared sense of purpose" (174); v1 "Establish an inspiring long-term vision" (161); v49 "Follow through on commitments" (159); v30 "Encourage sharing and learn from mistakes" (159); v59 "Use knowledge of conservation to inform decisions" (158); v3 "Identify what is happening to biodiversity" (158). Only seven items (again representing $10 \%$ of the framework) scored below the reference item score of 100 points (Establish budgets and manage costs), namely items v56, v26, v32, v13, v41, and v45 and $\mathrm{v} 4$ and these relate to personal challenge and reputation, planning, system appraisal, and use of people data (see Table S3). 


\section{Discussion}

This study presents empirical insights into conservation leadership using items derived from literature (Black et al., 2011; Bruyere, 2015; Dietz et al., 2004; Englefield et al., 2019; Kouzes \& Posner, 2007; Manolis et al., 2009). It is notable that the most highly rated (important) items relate to emerging issues in conservation leadership, namely dignity (Black, 2019; Straka et al., 2018), purpose (Black, Groombridge, \& Jones, 2011), vision (Bruyere, 2015), learning from mistakes (Catalano et al., 2021), and knowledge (Black \& Copsey 2014). The resulting six leadership constructs are reliable and valid, aligned with psychological evidence on leadership (Kilburg \& Donohue, 2011; Van Vugt et al., 2008). The model provides new insights plus confirmation of previously proposed concepts into a coherent framework of conservation leadership, which should encourage future studies on alternative samples of practitioners to confirm the predictive and discriminant validity of the factors.

The six-factor model (Table 7) confirms the negative impact of ego (Dietz et al., 2004), whilst emphasizing the importance of dignity in managing relationships in a multi-cultural environment often encountered in conservation (Mattson \& Clark, 2011; Straka et al., 2018). The Conservation Leadership Model emphasizes a focus on biodiversity, centred on encouraging dignity in followership, operational knowledge, pragmatic realism, vision, authenticity, and a purposefulness which is focused on what matters to biodiversity.

Table 7. Items in the 6 factors (order from Pattern Matrix with questionnaire number).

\section{Factor 1 "Serve Biodiversity Through Followers"}

13 Possess highly developed biological and/or operational skills appropriate to the program

49 Follow through on promises and commitments

3 Identify what is happening to, or affecting, biodiversity (populations, productivity, threats)

15 Empower staff to get the job done

60 Take the complexity of conservation issues into account when making decisions

5 Ensure that planning is flexible and make changes when required

7 Consider views of stakeholders and partners

59 Use knowledge of conservation to inform decisions

27 Be receptive to and seek out diverse opinions and alternative solutions

61 Care about the implications of decisions and how they relate to conservation success

50 Treat others with dignity and respect

16 Know people's strengths and channel their energy and passion to maximum effect

21 Have two-way communication meetings to discuss progress and goals

29 Enable staff to ask questions and challenge thinking

30 Encourage staff to share experiences, problems, ideas and learn from mistakes, without fear of criticism

31 Set high standards, giving a personal example of what is expected 


\section{Continued}

\section{Factor 2 "Understand Operational Work"}

8 Ensure planning starts from understanding current performance against intended program purpose*

26 Provide training on a just-in-time basis

18 Involve the people doing the work in data analysis, decisions and implementing changes

39 Establish a fundraising strategy

37 Understand what you can influence and avoid distraction by unsolvable problems outside your control

36 Allow people doing the work the freedom to experiment with methods to improve outcomes

10 Advocate good governance, particularly in large complex projects

19 Place responsibility and control of information in the hands of people who do the work

57 Look to others with more experience for feedback and discussion of ideas

12 Ensure consistency and alignment between plans, action on the ground and results 14 Prioritise the work by asking key questions and checking results

28 Understand risk and make suitable contingencies

32 Appraise the system and organisation of work, rather than people

4 Set clear, short-term goals

25 Give people the opportunity to ask for training

11 Be orientated towards "hands-on" management, working with staff.

23 Ensure managers lead and that they spend time with staff, listen to concerns and enable contributions

\section{Factor 3 "Sense of Reality"}

24 Expect the project (and its needs) to evolve through time

40 Determine relevant financial \& non-financial measures of performance

34 Make improvements based on biodiversity needs and process performance, not arbitrary targets

35 Recognise the difference between neglect and lack of capability (training, experience or resources)

38 Focus both internally and externally, understanding intra- and inter-organisational dynamics

9 Ensure that staff embrace project aims and culture (vision, understanding the system, goals)

2 Establish a shared sense of purpose throughout the team.

41 Base information, technology and resource plans on how they will help people's core work

22 Place an emphasis on personally clarifying, testing and establishing good understanding 


\section{Continued}

Factor 4 "Visionary Leadership"

56 Achieve a good reputation in conservation through personal successes

45 Determine whether data on staff, communities or society would be useful for the program

6 Measure performance against project aims

1 Establish a stable, inspiring and compelling shared long-term vision or "big picture"

62 Be aware of one's own strengths and weaknesses

Factor 5 "Authentic Engagement"

55 Have a clear personal philosophy of leadership

46 Talk about future trends (threats, socio-economics, funds, capacity) that influence how work gets done*

64 Recognise how one's own emotions and the feelings of the team influence decisions and actions

53 Support the work-related decisions that people make on their own 68 Let individual team members express their own skills and talents

67 Change leadership style and approach depending on what the situation requires 66 Identify potential leaders and support their development

52 Focus on what can be learned when things don't go as expected 48 Encourage people to have confidence in their own abilities

54 Publicly recognise and reward people who exemplify commitment to shared values 69 Encourage team members to form networks of support with colleagues

33 Manage morale, celebrate success, and creatively reward people's contributions

$70 \mathrm{Be}$ open to self-development by engaging support from those who are more skilled or experienced

17 Understand cultural differences and manage people's expectations and viewpoints sensitively

65 Provide expertise, guidance and support to the team

42 Create an attitude of co-operation with project partners, sharing information to improve effectiveness

Factor 6 "Purpose Before Ego"

43 Anticipate unexpected outcomes; integrate management flexibility alongside professional rigour

58 Communicate with conviction and demonstrate passion for the work that is being done

20 Ensure that an understanding of what matters to biodiversity steers the work that people do

63 Be willing to say "I don't know"

44 Be prepared to seek specialist advice and learn from external sources

The six factors are hereafter discussed in relation to existing leadership scales. 
Factor 1 (Serves Biodiversity through Followers) places an expectation that leaders make decisions based on conservation needs and an understanding of stakeholders (rather than, for example, finances, politics, personal reputation, or prestige). Understanding these expectations must also clearly involve intense engagement of colleagues and staff in that process. This reflects servant leadership (Barbuto Jr. \& Wheeler, 2006); Altruistic Calling, Emotional Healing, Wisdom, Persuasion and Stewardship. Although those specific terms do not appear in the items within Factor 1, all but two of the 16 items reflect moral, emotional, and relational dimensions of leadership (Reed et al., 2011) including, empowering staff, consideration of complexity, stakeholders, receptivity to opinions and challenge, dignity and respect, and learning from mistakes. Issues of ethics, dignity and respect have been shown to be critical in addressing cross-cultural issues (Straka et al., 2018; Black, 2019) and in supporting the role of women and minorities working professionally or as stakeholders in the sector (Byrne et al., 2018; Jones \& Solomon, 2019; Alvarez \& Lovera, 2016), both being areas of clear leadership responsibility. While the questionnaire items v13 (biological skills) and v5 (flexible planning) are less notably linked they nevertheless relate to how leaders create a positive "error management" culture (Hunter et al., 2011; Catalano et al., 2018; Catalano et al., 2019) and encourage innovation (Norbom \& Lopez, 2016). Conservation carries a strong vocational ethos and practitioners are advocates for species and ecosystems of concern (Black et al., 2011), so servanthood driven by a higher calling has relevance. The factor title "Serves biodiversity through followers" appears appropriate.

The leader must be purposed and committed to conserving biodiversity, an attitude of mind and a deliberate and transparent commitment. This factor highlights, however, that such individual commitment itself will not deliver the programme. What is needed is the input of the team and other stakeholders, and a channelling of their energy, skills and problem solving capacity. This is achieved by an approach which values those stakeholders and staff, keeps to promises made to those people, and thereby encourages their commitment.

Factor 2 (Understand Operational Work) is less obviously related to traditional notions of leadership, yet evidence of programme failures demonstrate that poor leadership is exhibited when the aspects of factor 2 are neglected. This factor has an operational focus (including potential organisational distractions) addressing planning, training, data analysis, fundraising, experimentation, governance, information control, contingencies, and goals. Shortfalls in these areas have seen failures with the po'ouli (Powell, 2008; Black \& Groombridge, 2010), the Christmas Island Pipistrelle (Martin et al., 2012), and the Yangtse river dolphin (Turvey, 2009). A suite of items relates to operational monitoring; drawing upon experience, hands-on management, and asking key questions to prioritise work, all of which relate to three fundamentals of motivation in self-determination theory (Ryan \& Deci, 2000), namely: goals (v4), autonomy (v18, v36, v19) and purpose (v8). This factor suggests that leadership integrity starts from the maxim "Understand operational work". 
These elements within Factor 2 represent an unusual area of competence which is not traditionally associated with western concepts of "leadership". The factor is comprised of a series of practical aspects within the leaders' role; planning, training, data analysis, fundraising. Nevertheless, these are clearly aligned to a leader's specific responsibilities, such as governance, risk management, delegation and prioritisation. Essentially the practical concerns of management cannot be separated from a leader's perspective on their organisation, and the relative importance placed on those aspects by that leader.

Factor 3 (Sense of Reality) relates to one of the most important competences in leadership, the ability that ensures that the leader takes a perspective that addresses real issues and challenges. This factor carries a number of items which express the concepts measured in Kalshoven et al.'s (2011) fairness scale, for example v35 (Recognise the difference between neglect and lack of capability) and v34 (Make improvements against biodiversity needs not arbitrary targets). Manipulative elements of the fairness scale do not appear in Factor 3 at all. Several of Factor 3 items (v24, v40, v34, v2, v9) consider constraints of time, cost, biodiversity needs, skills, resources and commitment of people which resonate with Conger and Kanungo's (1994) Environmental Sensitivity scale. Factor 3 appears to concern leadership effectiveness arising from a "Sense of Reality".

A leader with a sense of reality accepts changes in circumstances, utilises dissonance within the team, navigates organisational dynamics, and ensures that budgets are derived from an understanding of work capability, limitations and needs rather than driven from financial perspectives (i.e. the budget is designed from the requirements of conservation work, not the work being designed from the budget). This perspective resonates with significant leadership models such those proposed by Deming (1994) and Covey (1999).

Factor 4 (Visionary Leadership) examines the character and reputation of the leader alongside consideration of what is important for the organisation now and in the future. Self awareness is the key to a leader's ability to draw others in and get support (Covey, 1999). In terms of the collection of items, this is one of the simplest factors, reliant on the leader's ability to build a shared vision, supported by personal reputation and ability to define measurable aspects of that vision. Kouzes and Posner's (2007) principle of "inspiring a shared vision" is a close comparison. Factor 4 is not about simple communication of vision, as in Conger and Kanungo's (1994) Vision and Articulation scale. Instead, factor 4 involves a more nuanced consideration of vision as a distinguishing feature of transformational leadership (Carless, Wearing, \& Mann, 2000). Although Factor 4 is complex, it is perhaps best described as Visionary Leadership.

Factor 5 (Authentic Engagement) resonates with ethical leadership (Kalshoven et al., 2011) covering fairness, power, role clarification, people orientation, integrity, ethics, and sustainability, and partly with Participative Decision-Making (Arnold et al., 2000) including encouragement of ideas and group suggestions. Conservation commonly involves collaboration with external partners and communities (external advice, cooperate with partners, understand cul- 
tural differences, development by those more skilled, external trends). This factor embodies self-awareness, relational transparency, and moral perspective in authentic leadership (Neider \& Schriesheim, 2011). Effective leaders support and acknowledge individual's and team's achievements (Kouzes \& Posner, 2007; Carless et al., 2000) suggesting Factor 5 describes "Authentic engagement".

Engagement, relative to other aspects of conservation science and organisation, is poorly understood. It requires a particular personal perspective and set of behaviours as well as an ability to actively encourage types of behaviour in others, with both the personal and other-enabling aspects being necessary and complimentary. This results in a large variety of items appearing within the factor, yet the relationship between each of the items and their inclusion is important. Attempts to engage staff are undermined by failures in understanding cultural difference (v17), poor cooperation (v42), lack of celebration of successes (v33), blame rather than seeking to learn from failure (v52), lack of confidence of employees (v48) lack of interest by leaders in the work (v46), and lack of support form team member's suggestions and decisions (v53). Instead, application of "Authentic engagement" as fully represented in this factor is an important leadership principle.

Factor 6 (Purpose Before Ego) emphasizes the need for leaders' attention towards purpose (i.e. needs of species and ecosystems) and not themselves (ego or personal preferences). A leader should be open to review and change (v43) expecting that even their best plans can become impractical, misguided or no longer relevant. The leader's passionate message is about work, not themselves (v58). Work priorities should relate to what matters to biodiversity, overriding a leader's personal preference or professional bias (v20). Leaders must be humble enough (Kouzes \& Posner, 2007) to know that they do not have all the answers (v63, v44). In essence a leader places "Purpose before Ego".

Factor 6 is an important construct in that it takes leadership away from being dependent on the personality of the leader. By taking on the areas of competence within factor 6 , the individual leader is allowed to pursue effectiveness rather than preserve their own sense of self-worth. Self-worth is taken out of the arena and replaced by a focus on work. This resonates strongly with Kouzes and Posner's (2007) principle of leadership humility. Importantly the conservation leader needs this humble perspective when operating in the unpredictable working context encountered in conservation (Game et al., 2014) to enable anticipation and sensible response to influential but uncontrollable changes in the threats, and pressures on ecosystems and species of concern.

\section{Conclusion}

This study offers the first empirically-derived model of conservation leadership. This is an important step for the discipline of conservation science, since the discussion on leadership in the conservation sector still needs to mature in two dimensions. First, we need a better understanding of the important practices 


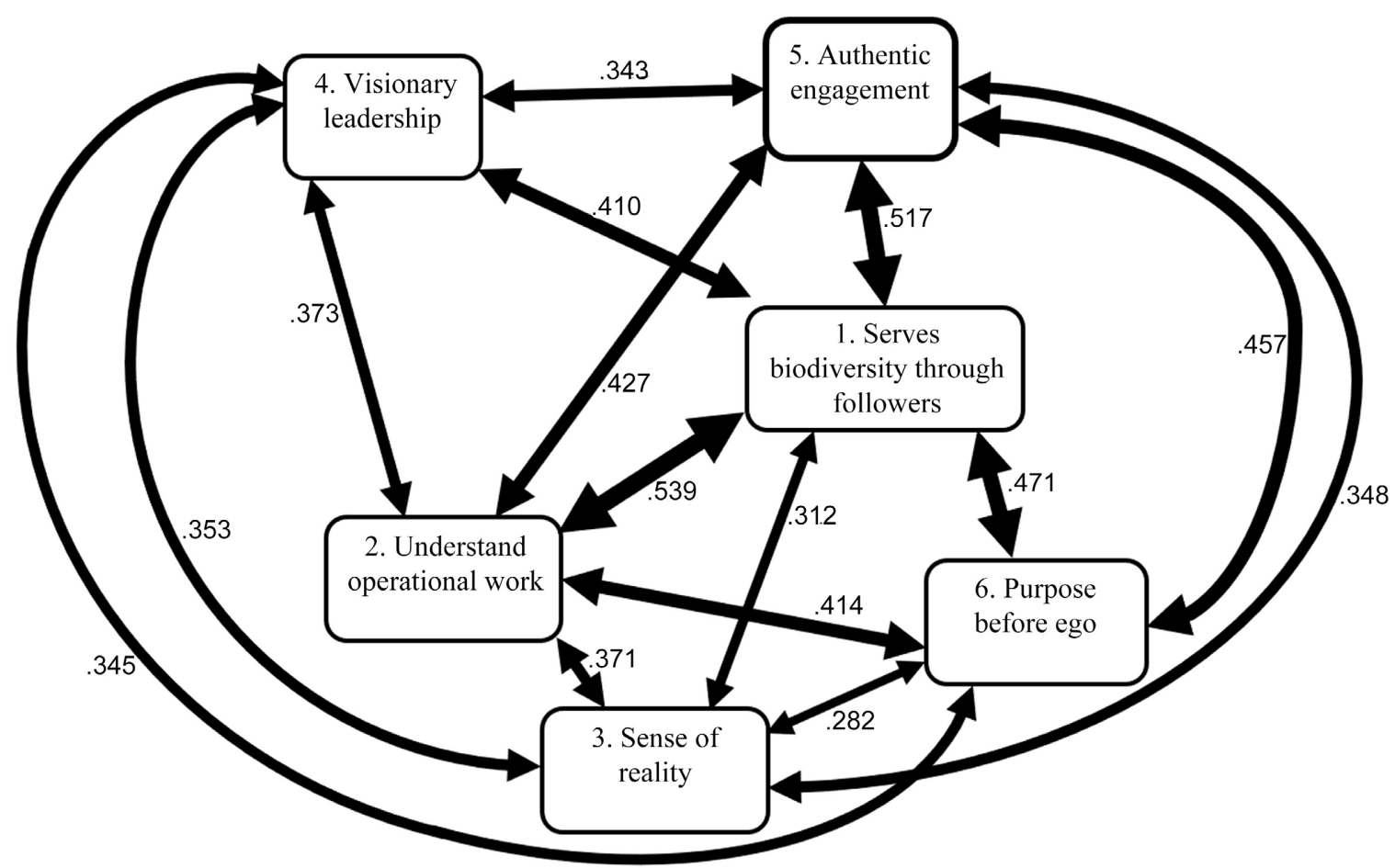

Figure 2. Conceptual model of the six factors of Conservation Leadership, showing correlations between factors (thickness of arrows emphasizes strengths of correlations).

that leaders must pursue to make conservation succeed. Second, we need to test whether those practices resonate with, and are congruent with, credible theories of leadership, psychology, and conservation science (rather than having practitioners simply relying on a collection of what is perceived to "make sense"). The presented six-factor model meets both of these criteria. Furthermore, it presents critical aspects which conservation professionals now need to explore in terms of their development to meet and lead conservation organisations, programmes and teams more effectively in the $21^{\text {st }}$ century. Conservation leaders need to be stretched to develop high performance organisations, in order to counteract the growing threats to biodiversity. This six-factor model of conservation leadership presents the demanding range of leadership practices that will be required to deliver that role.

The factors are notable in emphasizing authenticity and servanthood in leadership, alongside a pragmatic understanding of biodiversity needs and practical operations. The strongest inter-factor correlations (Figure 2) link "Serving Biodiversity through Followers" with both "Authentic Engagement" and "Understanding Operational Work". "Purpose before Ego" links strongly with "Authentic Engagement" and "Serving Biodiversity". Vision is a practical tool affirming a leader's personal understanding and expectations with the needs of conservation. A leader needs to put ego aside and instead focus on the realities of situations, rather than preconceptions or self-image, both being expectations which confirm assertions by Dietz et al. (2004) and Black et al. (2011). 
This study highlights the need for purposeful conservation leadership, focused on species and ecosystem needs, and dignified engagement of people through vision, empowerment and sound operational management. The six-factor model offers a memorable basis for leaders to consider their own competence. The model has potential as the basis for psychological assessment, or a curriculum for leadership training.

Conservation leaders face a collective and personal developmental challenge. They need to dispense with traditionally taught (or exemplified) behaviours relating to self-interest, ego, and hierarchy. Instead, conservation leaders must pursue new skills in influencing team development, stakeholder engagement, and managing improvement. At a personal level, conservation leaders need to work in a dignified, respectful way with communities, with their staff and with partner organisations yet must always retain advocacy for, and a focus upon, the needs of species and ecosystems of concern.

\section{Recommendations for Future Studies}

This study offers an important step in establishing appropriate understanding of leadership for the conservation sector. The research had some limitations in terms of the sample which necessarily needs to reflect the global nature of the sector. Improved samples are with proportionally more respondents specifically from Africa, Asia, South America and to a lesser extent Oceania and North America. Additionally a greater proportion of staff from Government Departments and multi-agency projects would be beneficial in future examination of the topic. An understanding of the effectiveness of conservation professionals in delivering these competencies within their role would give insight into the leadership capacity of this important sector of human endeavor.

\section{Acknowledgements}

Research support was provided by: W. Accouche, R. Black, J. Copsey, A. Diederichsen, H. Doulton, E. Englefield, H. Al Hikmani, T. Loffeld, C. Morais, J. Parker, A. Pungaliya, G. Randsley de Moura, S. Richards, D. Roberts, N. Webb, and R. Weckauf. Thanks to Durrell Wildlife Conservation Trust for access to the alumni network. The study was approved by the ethics procedures of the School of Psychology, University of Kent, following the code of conduct of the British Psychological Society (2010).

\section{Conflicts of Interest}

The author declares no conflicts of interest relating to publication of this paper.

\section{References}

Aarons, G. A., Ehrhart, M. G., \& Farahnak, L. R. (2014). The Implementation Leadership Scale (ILS): Development of a Brief Measure of Unit Level Implementation Leadership. Implementation Science, 9, 45. https://doi.org/10.1186/1748-5908-9-45 
Acheson, J. M. (2006). Institutional Failure in Resource Management. Annual Review of Anthropology, 35, 117-134. https://doi.org/10.1146/annurev.anthro.35.081705.123238

Alvarez, I., \& Lovera, S. (2016). New Times for Women and Gender Issues in Biodiversity Conservation and Climate Justice. Development, 59, 263-265. https://doi.org/10.1057/s41301-017-0111-Z

Arnold, J. A., Arad, S., Rhoades, J. A., \& Drasgow, F. (2000). The Empowering Leadership Questionnaire: The Construction and Validation of a New Scale for Measuring Leader Behaviors. Journal of Organizational Behavior, 21, 249-269.

http://www.jstor.org/stable/3100332 https://doi.org/10.1002/(SICI)1099-1379(200005)21:3<249::AID-JOB10>3.0.CO;2-\#

Barbuto Jr., J. E., \& Wheeler, D. W. (2006). Scale Development and Construct Clarification of Servant Leadership. Group \& Organization Management, 31, 300-326. https://doi.org/10.1177/1059601106287091

Barrett, P. T., \& Kline, P. (1981). The Observation to Variable Ratio in Factor Analysis. Personality Study and Group Behaviour, 1, 23-33.

Bass, B. M. (2000). The Future of Leadership in Learning Organizations. Journal of Leadership \& Organizational Studies, 7, 18-40. https://doi.org/10.1177/107179190000700302

Bass, B. M., Avolio, B. J., Jung, D. I., \& Berson, Y. (2003). Predicting Unit Performance by Assessing Transformational and Transactional Leadership. Journal of Applied Psychology, 88, 207. https://doi.org/10.1037/0021-9010.88.2.207

Bengston, D. N., \& Fan, D. P. (1999). An Innovative Method for Evaluating Strategic Goals in a Public Agency Conservation Leadership in the US Forest Service. Evaluation Review, 23, 77-100. https://doi.org/10.1177/0193841X9902300104

Bianco, M., Koss, R., \& Zischka, K. (2016). Empowering Emerging Leaders in Marine Conservation. Aquatic Conservation: Marine and Freshwater Ecosystems, 26, 225-236. https://doi.org/10.1002/aqc. 2650

Black, S. A., \& Copsey, J. A. (2014). Does Deming's "System of Profound Knowledge” Apply to Leaders of Biodiversity Conservation? Open Journal of Leadership, 3, 53-65. https://doi.org/10.4236/oj1.2014.32006

Black, S. A., Groombridge, J. J., \& Jones, C. G. (2011). Leadership and Conservation Effectiveness: Finding a Better Way to Lead. Conservation Letters, 4, 329-339. https://doi.org/10.1111/j.1755-263X.2011.00184.X

Black, S. A., Groombridge, J. J., \& Jones, C. G. (2013). Using Better Management Thinking to Improve Conservation Effectiveness. ISRN Biodiversity, 2013, Article ID: 784701. https://doi.org/10.1155/2013/784701

Black, S., \& Groombridge, J. I. M. (2010). Use of a Business Excellence Model to Improve Conservation Programs. Conservation Biology, 24, 1448-1458. https://doi.org/10.1111/j.1523-1739.2010.01562.x

Black, S. A. (2019). Psychological Models Relevant to Leadership in Wildlife Conservation. Open Journal of Leadership, 8, 114-141. https://doi.org/10.4236/ojl.2019.83007

British Psychological Society (2010). Code of Human Research Ethics. BPS. https://www.bps.org.uk/

Bruyere, B. L. (2015). Giving Direction and Clarity to Conservation Leadership. Conservation Letters, 8, 378-382. https://doi.org/10.1111/conl.12174

Bryman, A. (1992). Charisma and Leadership in Organizations. Sage Publications.

Byrne, M., Broadhurst, L., Leishman, M., \& Belov, K. (2018). Women in Conservation Science Making a Difference. Pacific Conservation Biology, 24, 209-214. 
https://doi.org/10.1071/PC18061

Carless, S. A., Wearing, A. J., \& Mann, L. (2000). A Short Measure of Transformational Leadership. Journal of Business and Psychology, 14, 389-405. https://link.springer.com/article/10.1023\%2FA\%3A1022991115523?LI=true https://doi.org/10.1023/A:1022991115523

Case, P., Evans, L. S., Fabinyi, M., Cohen, P. J., Hicks, C. C., Prideaux, M., \& Mills, D. J. (2015). Rethinking Environmental Leadership: The Social Construction of Leaders and Leadership in Discourses of Ecological Crisis, Development, and Conservation. Leadership, 11, 396-423. https://doi.org/10.1177/1742715015577887

Catalano, A. S., Jimmieson, N. L., \& Knight, A. T. (2021). Building Better Teams by Identifying Conservation Professionals Willing to Learn from Failure. Biological Conservation, 256, Article ID: 109069. https://doi.org/10.1016/j.biocon.2021.109069

Catalano, A. S., Lyons-White, J., Mills, M. M., \& Knight, A. T. (2019). Learning from Published Project Failures in Conservation. Biological Conservation, 238, Article ID: 108223. https://doi.org/10.1016/j.biocon.2019.108223

Catalano, A. S., Redford, K., Margoluis, R., \& Knight, A. T. (2018). Black Swans, Cognition, and the Power of Learning from Failure. Conservation Biology, 32, 584-596.

https://doi.org/10.1111/cobi.13045

Cattell, R. B. (1978). The Scientific Use of Factor Analysis in Behavioural and Life Sciences. Plenum Press. https://doi.org/10.1007/978-1-4684-2262-7

Cerny, C. A., \& Kaiser, H. F. (1977). A Study of a Measure of Sampling Adequacy for Factor-Analytic Correlation Matrices. Multivariate Behavioral Research, 12, 43-47. https://doi.org/10.1207/s15327906mbr1201_3

Clark, T. W., Reading, R. P., \& Clarke, A. L. (1994). Endangered Species Recovery: Finding the Lessons, Improving the Process. Island Press.

Comrey, A. L. (1973). A First Course in Factor Analysis. Academic Press.

Conger, J. A., \& Kanungo, R. N. (1994). Charismatic Leadership in Organizations: Perceived Behavioral Attributes and Their Measurement. Journal of Organizational Behavior, 15, 439-452. https://doi.org/10.1002/job.4030150508

Conger, J. A., Kanungo, R. N., Menon, S. T., \& Mathur, P. (1997). Measuring Charisma: Dimensionality and Validity of the Conger-Kanungo Scale of Charismatic Leadership. Canadian Journal of Administrative Sciences, 14, 290-301. https://doi.org/10.1111/j.1936-4490.1997.tb00136.x

Covey, S. R. (1999). Principle Centred Leadership (pp. 267-277). Simon \& Shuster UK Ltd.

Cox, M. (2016). The Pathology of Command and Control: A Formal Synthesis. Ecology and Society, 21, 33-40. https://doi.org/10.5751/ES-08698-210333

Cronbach, L. J. (1951). Coefficient Alpha and the Internal Structure of Tests. Psychometrika, 16, 297-334. https://doi.org/10.1007/BF02310555

Dancey, C. P., \& Reidy, J. (2011). Statistics without Maths for Psychology (5th ed., pp. 461-472). Pearson Education Limited.

Deming, W. E (1994). The New Economics for Industry, Education, Government. MIT Center for Advanced Engineering Study.

Dietz, J. M., Aviram, R., Bickford, S., Douthwaite, K., Goodstine, A., Izursa, J. L., \& Parker, K. (2004). Defining Leadership in Conservation: A View from the Top. Conservation Biology, 18, 274-278. https://doi.org/10.1111/j.1523-1739.2004.00554.x

Dziuban, C. D., \& Shirkey, E. C. (1974). When Is a Correlation Matrix Appropriate for 
Factor Analysis? Some Decision Rules. Psychological Bulletin, 81, 358-361. https://doi.org/10.1037/h0036316

Englefield, E., Black, S. A., Copsey, J. A., \& Knight, A. T. (2019). Interpersonal Competencies Define Effective Conservation Leadership. Biological Conservation, 235, 18-26. https://doi.org/10.1016/j.biocon.2019.03.043

Evans, L. S., Hicks, C. C., Cohen, P. J., Case, P., Prideaux, M., \& Mills, D. J. (2015). Understanding Leadership in the Environmental Sciences. Ecology and Society, 20, 50-60. https://doi.org/10.5751/ES-07268-200150

Game, E. T., Meijaard, E., Sheil, D., \& McDonald-Madden, E. (2014). Conservation in a Wicked Complex World; Challenges and Solutions. Conservation Letters, 7, 271-277. https://doi.org/10.1111/conl.12050

Gutierrez, N. L., Hilborn, R., \& Defeo, O. (2011). Leadership, Social Capital and Incentives Promote Successful Fisheries. Nature, 470, 386-389.

https://doi.org/10.1038/nature09689

Harman, H. H. (1967). Modern Factor Analysis (2nd ed.). University of Chicago Press.

Haslam, S. A., \& Reicher, S. D. (2016). Rethinking the Psychology of Leadership: From Personal Identity to Social Identity. Daedelus, Journal of the American Academy of Arts and Sciences, 145, 21-34. https://doi.org/10.1162/DAED a 00394

Haubold, E. M. (2012). Using Adaptive Leadership Principles in Collaborative Conservation with Stakeholders to Tackle a Wicked Problem: Imperilled Species Management in Florida. Human Dimensions of Wildlife, 17, 344-356.

https://doi.org/10.1080/10871209.2012.709308

Higgs, M., \& Rowland, D. (2011). What Does It Take to Implement Change Successfully? A Study of the Behaviors of Successful Change Leaders. The Journal of Applied Behavioral Science, 47, 309-335. https://doi.org/10.1177/0021886311404556

Holling, C. S., \& Meffe, G. K. (1996). Command and Control and the Pathology of Natural Resource Management. Conservation Biology, 10, 328-337. https://doi.org/10.1046/j.1523-1739.1996.10020328.x

House, R. J., Hanges, P. J., Ruiz-Quintanilla, S. A., Dorfman, P. W., Javidan, M., Dickson, M., \& Gupta, V. (1999). Cultural Influences on Leadership and Organizations: Project GLOBE. Advances in Global Leadership, 1, 171-233.

Hunter, S. T., Tate, B. W., Dzieweczynski, J. L., \& Bedell-Avers, K. E. (2011). Leaders Make Mistakes: A Multilevel Consideration of Why. The Leadership Quarterly, 22, 239-258. https://doi.org/10.1016/j.leaqua.2011.02.001

Imperial, M. T., Ospina, S., Johnston, E., O’Leary, R., Thomsen, J., Williams, P., \& Johnson, S. (2016). Understanding Leadership in a World of Shared Problems: Advancing Network Governance in Large Landscape Conservation. Frontiers in Ecology and the Environment, 14, 126-134. https://doi.org/10.1002/fee.1248

Jones, M. S., \& Solomon, J. (2019). Challenges and Supports for Women Conservation Leaders. Conservation Science and Practice, 1, e36. https://doi.org/10.1111/csp2.36

Kalshoven, K., Den Hartog, D. N., \& De Hoogh, A. H. B. (2011). Ethical Leadership at Work Questionnaire (ELW): Development and Validation of a Multidimensional Measure. The Leadership Quarterly, 22, 51-69. https://doi.org/10.1016/j.leaqua.2010.12.007

Khuntia, R., \& Suar, D. (2004). A Scale to Assess Ethical Leadership of Indian Private and Public Sector Managers. Journal of Business Ethics, 49, 13-26. https://doi.org/10.1023/B:BUSI.0000013853.80287.da

Kilburg, R. R., \& Donohue, M. D. (2011). Toward a “Grand Unifying Theory” of Leadership: Implications for Consulting Psychology. Consulting Psychology Journal: Practice 
and Research, 63, 6. https://doi.org/10.1037/a0023053

Kim, J., \& Mueller, L. W. (1978). Factor Analysis: Statistical Methods and Practical Issues. Sage University Paper Series on Quantitative Applications in the Social Sciences, 07-014, Sage Publications.

Kouzes, J. M., \& Posner, B. Z. (2007). The Leadership Challenge (4th ed.). Jossey-Bass Publishers.

Lodge, M. (1981). Magnitude Scaling: Quantitative Measurement of Opinions. Sage University Paper Series on Quantitative Applications in the Social Sciences, Series No 07-001. Beverley Hills and London; Sage Publications.

Manolis, J. C., Chan, K., Finkelstein, M., Stephens, S., Nelson, C., Grant, J., \& Dombeck, M. (2009). Leadership: A New Frontier in Conservation Science. Conservation Biology, 23, 879-886. https://doi.org/10.1111/j.1523-1739.2008.01150.x

Martin, T. G., Nally, S., Burbidge, A. A., Arnall, S., Garnett, S. T., Hayward, M. W., Lumsden, L. F., Menkhorst, P., McDonald-Madden, E., \& Possingham, H. P. (2012). Acting Fast Helps Avoid Extinction. Conservation Letters, 5, 274-280. https://doi.org/10.1111/j.1755-263X.2012.00239.x

Mattson, D. J., \& Clark, S. G. (2011). Human Dignity in Concept and Practice. Policy Sciences, 44, 303-320. https://doi.org/10.1007/s11077-010-9124-0

McCallum, S., \& O'Connell, D. (2009). Social Capital and Leadership Development. Leadership \& Organization Development Journal, 30, 152-165. https://doi.org/10.1108/01437730910935756

Narens, L. (1996). A Theory of Ratio Magnitude Estimation. Journal of Mathematical Psychology, 40, 109-129. https://doi.org/10.1006/jmps.1996.0011

Neider, L. L., \& Schriesheim, C. A. (2011). The Authentic Leadership Inventory (ALI): Development and Empirical Tests. The Leadership Quarterly, 22, 1146-1164. https://doi.org/10.1016/j.leaqua.2011.09.008

Norbom, H. M., \& Lopez, P. D. (2016). Leadership and Innovation: Informal Power and Its Relationship to Innovative Culture. Journal of Leadership Studies, 10, 18-31. https://doi.org/10.1002/jls.21430

Nunnally, J. C. (1967). Psychometric Theory. McGraw-Hill.

Posner, B. Z. (2016). Investigating the Reliability and Validity of the Leadership Practices Inventory ${ }^{\circledR}$. Administrative Sciences, 6, 17. https://doi.org/10.3390/admsci6040017

Powell, A. (2008). The Race to Save the World's Rarest Bird: The Discovery and Death of the Po'ouli. Stackpole Books.

Quammen, D. (2012). The Song of the Dodo: Island Biogeography in an Age of Extinctions. Random House.

Reed, L. L., Vidaver-Cohen, D., \& Colwell, S. R. (2011). A New Scale to Measure Executive Servant Leadership: Development, Analysis, and Implications for Research. Journal of Business Ethics, 101, 415-434. https://doi.org/10.1007/s10551-010-0729-1

Rousseau, D. M., Sitkin, S. B., Burt, R. S., \& Camerer, C. (1998). Not So Different after All: A Cross-Discipline View of Trust. Academy of Management Review, 23, 393-404. https://doi.org/10.5465/amr.1998.926617

Rummel, R. J. (1970). Applied Factor Analysis. Northwestern Univ. Press.

Ryan, R. M., \& Deci, E. L. (2000). Self-Determination Theory and the Facilitation of Intrinsic Motivation, Social Development, and Well-Being. American Psychologist, 55, 68. https://doi.org/10.1037/0003-066X.55.1.68 
Seddon, J. (2003). Freedom from Command and Control. Vanguard Press.

Sjölander-Lindqvist, A., Johansson, M., \& Sandström, C. (2015). Individual and Collective Responses to Large Carnivore Management: The Roles of Trust, Representation, Knowledge Spheres, Communication and Leadership. Wildlife Biology, 21, 175-185. https://doi.org/10.2981/wlb.00065

Straka, T. M., Bal, P., Corrigan, C., Di Fonzo, M. M., \& Butt, N. (2018). Conservation Leadership Must Account for Cultural Differences. Journal for Nature Conservation, 43, 111-116. https://doi.org/10.1016/j.jnc.2018.03.003

Sutton, A. E. (2015). Leadership and Management Influences the Outcome of Wildlife Reintroduction Programs: Findings from the Sea Eagle Recovery Project. PeerJ, 3, e1012. https://doi.org/10.7717/peerj.1012

Thite (1999). Identifying Key Characteristics of Technical Project Leadership. Leadership \& Organization Development Journal, 20, 253-261. https://doi.org/10.1108/01437739910287126

Thurstone, L. L. (1947). Multiple Factor Analysis. University of Chicago Press.

Turvey, S. (2009). Witness to Extinction: How We Failed to Save the Yangtze River Dolphin. Oxford University Press.

Van Vugt, M., Hogan, R., \& Kaiser, R. B. (2008). Leadership, Followership, and Evolution: Some Lessons from the Past. American Psychologist, 63, 182. https://doi.org/10.1037/0003-066X.63.3.182

Webb, S. A., Breyer, B., Halladay, M., \& Walker, S. (2020). A Framework for Conceptualising Leadership in Conservation. Oryx, 1-7. https://doi.org/10.1017/S0030605320000629 


\section{Supplementary}

Table S1. (a-f) Unifactorial tests of each factor (6 pages).

\begin{tabular}{lc}
\hline & Component \\
\cline { 2 - 2 } & 1 \\
\hline $\begin{array}{l}\text { Be receptive to and seek out diverse opinions and alternative solutions } \\
\text { Know people's strengths and channel their energy and passion to } \\
\text { maximum effect }\end{array}$ & 0.948 \\
$\begin{array}{l}\text { Ensure that planning is flexible and make changes when required } \\
\text { Empower staff to get the job done }\end{array}$ & 0.945 \\
$\begin{array}{l}\text { Care about the implications of decisions and how they relate to } \\
\text { conservation success }\end{array}$ & 0.941 \\
$\begin{array}{l}\text { Have two-way communication meetings to discuss progress and goals } \\
\text { Encourage staff to share experiences, problems, ideas and learn from } \\
\text { mistakes, without fear of criticism }\end{array}$ & 0.925 \\
$\begin{array}{l}\text { Follow through on promises and commitments } \\
\begin{array}{l}\text { Take the complexity of conservation issues into account when making } \\
\text { decisions }\end{array}\end{array}$ & 0.917 \\
$\begin{array}{l}\text { Enable staff to ask questions and challenge thinking } \\
\text { Use knowledge of conservation to inform decisions }\end{array}$ & 0.915 \\
$\begin{array}{l}\text { Treat others with dignity and respect } \\
\begin{array}{l}\text { Identify what is happening to, or affecting, biodiversity (populations, } \\
\text { productivity, threats) }\end{array}\end{array}$ \\
$\begin{array}{l}\text { Set high standards, giving a personal example of what is expected } \\
\text { the program }\end{array}$ & 0.993 \\
\hline
\end{tabular}

Extraction Method: Principal Component Analysis. ${ }^{\mathrm{a}} 1$ components extracted.

\begin{tabular}{|c|c|c|c|c|c|c|}
\hline \multirow{2}{*}{$\begin{array}{c}\text { Total } \\
\text { Component }\end{array}$} & \multicolumn{3}{|c|}{$\begin{array}{l}\text { Variance Explained } \\
\text { Initial Eigenvalues }\end{array}$} & \multicolumn{3}{|c|}{$\begin{array}{c}\text { Extraction Sums of Squared } \\
\text { Loadings }\end{array}$} \\
\hline & Total & $\begin{array}{c}\% \text { of } \\
\text { Variance }\end{array}$ & Cumul. \% & Total & $\begin{array}{c}\% \text { of } \\
\text { Variance }\end{array}$ & Cumul. \% \\
\hline 1 & 13.017 & 81.354 & 81.354 & 13.017 & 81.354 & 81.354 \\
\hline 2 & 0.641 & 4.006 & 85.360 & & & \\
\hline 3 & 0.542 & 3.385 & 88.745 & & & \\
\hline 4 & 0.312 & 1.951 & 90.696 & & & \\
\hline 5 & 0.271 & 1.696 & 92.392 & & & \\
\hline 6 & 0.225 & 1.408 & 93.800 & & & \\
\hline 7 & 0.216 & 1.351 & 95.152 & & & \\
\hline 8 & 0.168 & 1.051 & 96.202 & & & \\
\hline
\end{tabular}




\section{Continued}

\begin{tabular}{llll}
\hline 9 & 0.145 & 0.907 & 97.110 \\
10 & 0.121 & 0.754 & 97.864 \\
11 & 0.086 & 0.538 & 98.401 \\
12 & 0.083 & 0.519 & 98.920 \\
13 & 0.068 & 0.423 & 99.344 \\
14 & 0.052 & 0.327 & 99.670 \\
15 & 0.028 & 0.177 & 99.847 \\
16 & 0.024 & 0.153 & 100.000 \\
\hline
\end{tabular}

\begin{tabular}{ccc}
\hline \multicolumn{3}{c}{ KMO and Bartlett's Test } \\
\hline Kaiser-Meyer-Olkin Measure of Sampling Adequacy. & 0.930 \\
Bartlett's Test of Sphericity & Approx. Chi-Square & 1489.653 \\
& Df & 120 \\
& Sig. & 0.000 \\
\hline
\end{tabular}

b) Factor 2 Component Matrix

Component

1

Set clear, short-term goals

0.934

Prioritise the work by asking key questions and checking results

Allow people freedom to experiment with methods to improve outcomes

Advocate good governance, particularly in large complex projects

0.916

Involve people doing the work in data analysis, decisions \& implementing changes

Understand risk and make suitable contingencies

0.906

Understand what you can influence and avoid distraction by unsolvable problems outside your control

0.901

Ensure managers lead and that they spend time with staff, listen to concerns and enable contributions

Place responsibility \& control of information in the hands of people doing the work

Look to others with more experience for feedback and discussion of ideas

0.895

Ensure consistency and alignment between plans, action on the ground \& results

Be orientated towards "hands-on" management, working with staff.

Ensure that planning is flexible and make changes when required

0.834

Give people the opportunity to ask for training

Establish a fundraising strategy

Appraise the system and organisation of work, rather than people

Provide training on a just-in-time basis

Extraction Method: Principal Component Analysis. ${ }^{a} 1$ components extracted. 


\begin{tabular}{|c|c|c|c|c|c|c|}
\hline \multirow{2}{*}{$\begin{array}{c}\text { Total } \\
\text { Component }\end{array}$} & \multicolumn{3}{|c|}{$\begin{array}{l}\text { Variance Explained } \\
\text { Initial Eigenvalues }\end{array}$} & \multicolumn{3}{|c|}{$\begin{array}{l}\text { Extraction Sums of } \\
\text { Squared Loadings }\end{array}$} \\
\hline & Total & $\begin{array}{c}\% \text { of } \\
\text { Variance }\end{array}$ & Cumul. \% & Total & $\begin{array}{c}\% \text { of } \\
\text { Variance }\end{array}$ & Cumul. \% \\
\hline 1 & 12.488 & 73.458 & 73.458 & 12.488 & 73.458 & 73.458 \\
\hline 2 & 0.947 & 5.572 & 79.031 & & & \\
\hline 3 & 0.716 & 4.209 & 83.240 & & & \\
\hline 4 & 0.587 & 3.450 & 86.690 & & & \\
\hline 5 & 0.441 & 2.592 & 89.282 & & & \\
\hline 6 & 0.337 & 1.984 & 91.265 & & & \\
\hline 7 & 0.274 & 1.610 & 92.876 & & & \\
\hline 8 & 0.247 & 1.453 & 94.329 & & & \\
\hline 9 & 0.207 & 1.215 & 95.544 & & & \\
\hline 10 & 0.162 & 0.952 & 96.496 & & & \\
\hline 11 & 0.142 & 0.836 & 97.333 & & & \\
\hline 12 & 0.114 & 0.672 & 98.005 & & & \\
\hline 13 & 0.104 & 0.611 & 98.616 & & & \\
\hline 14 & 0.094 & 0.553 & 99.168 & & & \\
\hline 15 & 0.057 & 0.337 & 99.505 & & & \\
\hline 16 & 0.053 & 0.314 & 99.819 & & & \\
\hline 17 & 0.031 & 0.181 & 100.000 & & & \\
\hline
\end{tabular}

\section{KMO and Bartlett's Test}

Kaiser-Meyer-Olkin Measure of Sampling Adequacy.

0.935

Approx. Chi-Square

1306.343

Bartlett's Test of Sphericity df

136

Sig.

0.000

c) Factor 3 Component Matrix

Component

Establish a shared sense of purpose throughout the team.

0.932

Ensure that staff embrace project aims and culture (vision, understanding the system, goals)

0.926

Make improvements based on biodiversity needs and process performance, not arbitrary targets

Focus both internally and externally, understanding intra- and inter-organisational dynamics

Recognise the difference between neglect and lack of capability (training, experience or resources)

Place an emphasis on personally clarifying, testing and establishing good understanding 


\section{Continued}

Expect the project (and its needs) to evolve through time

Base information, technology and resource plans on how they will help people's core work

Determine relevant financial \& non-financial measures of performance

Extraction Method: Principal Component Analysis. ${ }^{a} 1$ components extracted.

\begin{tabular}{|c|c|c|c|c|c|c|}
\hline \multicolumn{7}{|c|}{ Total Variance Explained } \\
\hline \multirow{2}{*}{ Component } & \multicolumn{3}{|c|}{ Initial Eigenvalues } & \multicolumn{3}{|c|}{$\begin{array}{l}\text { Extraction Sums of } \\
\text { Squared Loadings }\end{array}$} \\
\hline & Total & $\begin{array}{c}\% \text { of } \\
\text { Variance }\end{array}$ & Cumul \% & Total & $\begin{array}{c}\% \text { of } \\
\text { Variance }\end{array}$ & Cumul \% \\
\hline 1 & 7.049 & 78.325 & 78.325 & 7.049 & 78.325 & 78.325 \\
\hline 2 & 0.470 & 5.221 & 83.546 & & & \\
\hline 3 & 0.374 & 4.152 & 87.698 & & & \\
\hline 4 & 0.336 & 3.731 & 91.429 & & & \\
\hline 5 & 0.287 & 3.188 & 94.617 & & & \\
\hline 6 & 0.149 & 1.653 & 96.270 & & & \\
\hline 7 & 0.134 & 1.494 & 97.764 & & & \\
\hline 8 & 0.110 & 1.218 & 98.982 & & & \\
\hline 9 & 0.092 & 1.018 & 100.000 & & & \\
\hline
\end{tabular}

Extraction Method: Principal Component Analysis.

\section{KMO and Bartlett's Test}

Kaiser-Meyer-Olkin Measure of Sampling Adequacy.

0.920

Approx. Chi-Square

Bartlett's Test of Sphericity

df

Sig.

\section{d) Factor 4 Component Matrix}

Component

1

Determine whether data on staff, communities or society would be useful for the program

0.891

Be aware of one's own strengths and weaknesses

0.852

Measure performance against project aims

0.845

Establish a stable, inspiring and compelling shared long-term vision or "big picture"

Achieve a good reputation in conservation through personal successes

0.769

Extraction Method: Principal Component Analysis. ${ }^{\mathrm{a}} 1$ components extracted. 
Total Variance Explained

\begin{tabular}{ccccccc}
\hline & \multicolumn{3}{c}{ Initial Eigenvalues } & \multicolumn{2}{c}{ Extraction Sums of Squared Loadings } \\
\cline { 2 - 6 } Component & Total & $\begin{array}{c}\text { \% of } \\
\text { Variance }\end{array}$ & Cumulative \% & Total & $\begin{array}{c}\text { \% of } \\
\text { Variance }\end{array}$ & Cumul \% \\
\hline 1 & 3.526 & 70.518 & 70.518 & 3.526 & 70.518 & 70.518 \\
2 & 0.528 & 10.568 & 81.086 & & \\
3 & 0.474 & 9.477 & 90.563 & & \\
4 & 0.287 & 5.733 & 96.297 & & & \\
5 & 0.185 & 3.703 & 100.000 & & & \\
\hline
\end{tabular}

Extraction Method: Principal Component Analysis.

\section{KMO and Bartlett's Test}

Kaiser-Meyer-Olkin Measure of Sampling Adequacy.

0.824

Approx. Chi-Square

172.921

Bartlett's Test of Sphericity

df

10

Sig.

0.000

e) Factor 5 Component Matrix ${ }^{\mathrm{a}}$

Component

1

Change leadership style and approach depending on what the situation requires

Encourage people to have confidence in their own abilities

Manage morale, celebrate success, and creatively reward people's contributions

0.910

Be open to self-development by engaging support from those who are more skilled or experienced

0.902

Create an attitude of co-operation with project partners, sharing information to improve effectiveness

Support the work-related decisions that people make on their own

Publicly recognise and reward people who exemplify commitment to shared values

Provide expertise, guidance and support to the team

0.886

Understand cultural differences \& manage expectations \& viewpoints sensitively

Encourage team members to form networks of support with colleagues

Let individual team members express their own skills and talents

Recognise how one's emotions \& team's feelings influence decisions \& actions 


\section{Continued}

Focus on what can be learned when things don't go as expected

Talk about future trends (threats, socio-economics, funding, capacity) that will influence how the work gets done

Have a clear personal philosophy of leadership

Extraction Method: Principal Component Analysis. ${ }^{\mathrm{a}} 1$ components extracted.

\begin{tabular}{|c|c|c|c|c|c|c|}
\hline \multirow{2}{*}{$\begin{array}{c}\text { Total } \\
\text { Component }\end{array}$} & \multicolumn{3}{|c|}{$\begin{array}{l}\text { Variance Explained } \\
\text { Initial Eigenvalues }\end{array}$} & \multicolumn{3}{|c|}{$\begin{array}{l}\text { Extraction Sums of } \\
\text { Squared Loadings }\end{array}$} \\
\hline & Total & $\begin{array}{c}\% \text { of } \\
\text { Variance }\end{array}$ & Cumul \% & Total & $\begin{array}{c}\% \text { of } \\
\text { Variance }\end{array}$ & Cumul. \% \\
\hline 1 & 12.424 & 77.652 & 77.652 & 12.424 & 77.652 & 77.652 \\
\hline 2 & 0.820 & 5.122 & 82.774 & & & \\
\hline 3 & 0.552 & 3.449 & 86.223 & & & \\
\hline 4 & 0.463 & 2.892 & 89.115 & & & \\
\hline 5 & 0.305 & 1.907 & 91.022 & & & \\
\hline 6 & 0.249 & 1.557 & 92.579 & & & \\
\hline 7 & 0.224 & 1.401 & 93.980 & & & \\
\hline 8 & 0.190 & 1.185 & 95.164 & & & \\
\hline 9 & 0.173 & 1.083 & 96.247 & & & \\
\hline 10 & 0.125 & 0.783 & 97.030 & & & \\
\hline 11 & 0.124 & 0.777 & 97.807 & & & \\
\hline 12 & 0.103 & 0.643 & 98.451 & & & \\
\hline 13 & 0.098 & 0.615 & 99.066 & & & \\
\hline 14 & 0.070 & 0.437 & 99.503 & & & \\
\hline 15 & 0.044 & 0.277 & 99.780 & & & \\
\hline 16 & 0.035 & 0.220 & 100.000 & & & \\
\hline
\end{tabular}

\section{KMO and Bartlett's Test}

Kaiser-Meyer-Olkin Measure of Sampling Adequacy.

Approx. Chi-Square

1314.079

Bartlett's Test of Sphericity $\mathrm{df}$

120

Sig.

0.000

\begin{tabular}{|c|c|}
\hline \multirow{2}{*}{ f) Factor 6 Component Matrix ${ }^{a}$} & Component \\
\hline & 1 \\
\hline $\begin{array}{l}\text { Anticipate unexpected outcomes; integrate management flexibility } \\
\text { alongside professional rigour }\end{array}$ & 0.908 \\
\hline Be prepared to seek specialist advice and learn from external sources & 0.889 \\
\hline $\begin{array}{l}\text { Communicate with conviction and demonstrate passion for the work that } \\
\text { is being done }\end{array}$ & 0.864 \\
\hline
\end{tabular}




\section{Continued}

Ensure that an understanding of what matters to biodiversity steers the work that people do

Be willing to say "I don't know"

Extraction Method: Principal Component Analysis. ${ }^{a} 1$ components extracted.

\begin{tabular}{|c|c|c|c|c|c|c|}
\hline \multicolumn{7}{|c|}{ Total Variance Explained } \\
\hline \multirow{2}{*}{ Component } & \multicolumn{3}{|c|}{ Initial Eigenvalues } & \multicolumn{3}{|c|}{$\begin{array}{l}\text { Extraction Sums of } \\
\text { Squared Loadings }\end{array}$} \\
\hline & Total & $\begin{array}{c}\% \text { of } \\
\text { Variance }\end{array}$ & Cumulative \% & Total & $\begin{array}{c}\% \text { of } \\
\text { Variance }\end{array}$ & Cumulative \% \\
\hline 1 & 3.797 & 75.932 & 75.932 & 3.797 & 75.932 & 75.932 \\
\hline 2 & 0.484 & 9.682 & 85.614 & & & \\
\hline 3 & 0.292 & 5.833 & 91.447 & & & \\
\hline 4 & 0.233 & 4.669 & 96.116 & & & \\
\hline 5 & 0.194 & 3.884 & 100.000 & & & \\
\hline
\end{tabular}

Extraction Method: Principal Component Analysis.

\begin{tabular}{ccc}
\hline \multicolumn{3}{c}{ KMO and Bartlett's Test } \\
\hline Kaiser-Meyer-Olkin Measure of Sampling Adequacy. & 0.868 \\
Bartlett's Test of Sphericity & Approx. Chi-Square & 213.736 \\
& df & 10 \\
& Sig. & 0.000 \\
\hline
\end{tabular}

Table S2. Reliability of each of the six factor scales as taken from the unifactorial sets in Table S1.

\begin{tabular}{crrl}
\hline Factor & Cronbach's Alpha & Number of Items & \multicolumn{1}{c}{ Items in the factor } \\
\hline 1 & 0.984 & 16 items & $13,49,3,15,60,5,7,59,27,61,50,16,21,29,30,31$ \\
2 & 0.976 & 17 items & $8,26,18,39,37,36,10,19,57,12,14,28,32,4,25,11,23$ \\
3 & 0.962 & 9 items & $24,40,34,35,38,9,2,41,22$ \\
4 & 0.880 & 5 items & $56,45,6,1,62$ \\
5 & 0.979 & 16 items & $55,46,64,53,68,67,66,52,48,54,69,33,70,17,65,42$ \\
6 & 0.920 & 5 items & $43,58,20,63,44$ \\
\hline
\end{tabular}


Table S3. Item importance scores (in brackets, Range $=67$ to 187) derived from the exponent of the mean of natural logs (all respondent scores per item), excluding reference item v0 (100) and the low loading item v47 which was removed from the factor analysis. Median importance score 130 points, with the distribution of scores tending towards normal (see Figure S1).
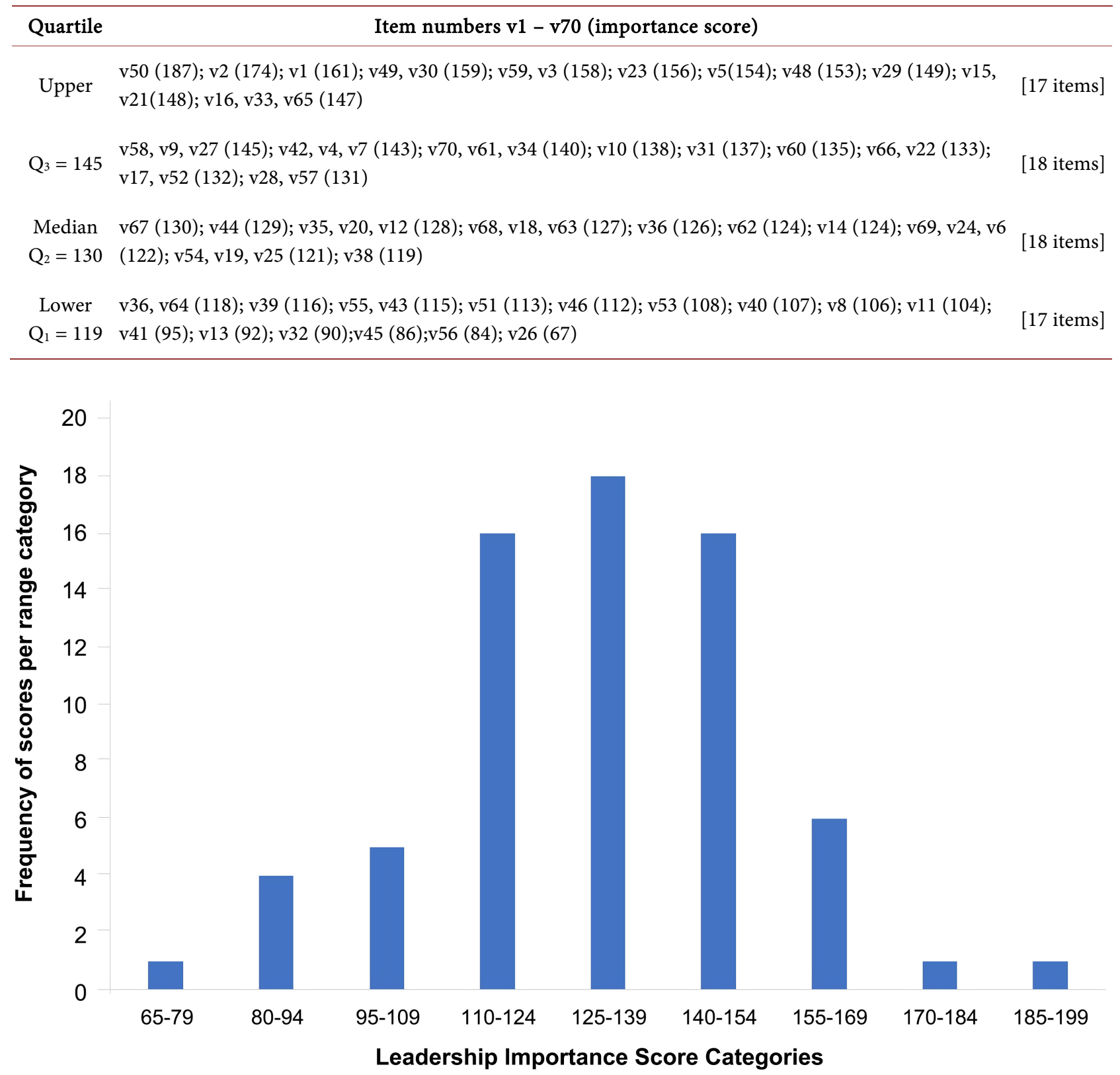

Figure S1. Frequency histogram of the ration scale of leadership importance sores for the 68 items derived from scale scores from all respondents (calculated as the exponent of the mean of natural logs of respondent scores) demonstrating the expected tendency towards a normal distribution (Lodge, 1981). 